\title{
Functional analysis of sense organ specification in the Tribolium castaneum larva reveals divergent mechanisms in insects
}

\author{
Marleen Klann ${ }^{1,2 \dagger}$, Magdalena Ines Schacht ${ }^{1 \dagger}$, Matthew Alan Benton ${ }^{3}$ and Angelika Stollewerk ${ }^{1 *}$ (D)
}

\begin{abstract}
Insects and other arthropods utilise external sensory structures for mechanosensory, olfactory, and gustatory reception. These sense organs have characteristic shapes related to their function, and in many cases are distributed in a fixed pattern so that they are identifiable individually. In Drosophila melanogaster, the identity of sense organs is regulated by specific combinations of transcription factors. In other arthropods, however, sense organ subtypes cannot be linked to the same code of gene expression. This raises the questions of how sense organ diversity has evolved and whether the principles underlying subtype identity in D. melanogaster are representative of other insects. Here, we provide evidence that such principles cannot be generalised, and suggest that sensory organ diversification followed the recruitment of sensory genes to distinct sensory organ specification mechanism.
\end{abstract}

Results: We analysed sense organ development in a nondipteran insect, the flour beetle Tribolium castaneum, by gene expression and RNA interference studies. We show that in contrast to D. melanogaster, T. castaneum sense organs cannot be categorised based on the expression or their requirement for individual or combinations of conserved sense organ transcription factors such as cut and pox neuro, or members of the Achaete-Scute (TC ASH, Tc asense), Atonal (Tc atonal, Tc cato, Tc amos), and neurogenin families (Tc tap). Rather, our observations support an evolutionary scenario whereby these sensory genes are required for the specification of sense organ precursors and the development and differentiation of sensory cell types in diverse external sensilla which do not fall into specific morphological and functional classes.

Conclusions: Based on our findings and past research, we present an evolutionary scenario suggesting that sense organ subtype identity has evolved by recruitment of a flexible sensory gene network to the different sense organ specification processes. A dominant role of these genes in subtype identity has evolved as a secondary effect of the function of these genes in individual or subsets of sense organs, probably modulated by positional cues.

Keywords: Tribolium castaneum, Sense organ development, Sense organ subtypes, Evolution, Gene expression, RNA interference

\footnotetext{
* Correspondence: a.stollewerk@qmul.ac.uk

${ }^{\dagger}$ Marleen Klann and Magdalena Ines Schacht contributed equally to this work.

'School of Biological and Chemical Sciences, Queen Mary University of London, Mile End Road, London E1 4NS, UK

Full list of author information is available at the end of the article
}

(c) The Author(s). 2021 Open Access This article is licensed under a Creative Commons Attribution 4.0 International License, which permits use, sharing, adaptation, distribution and reproduction in any medium or format, as long as you give appropriate credit to the original author(s) and the source, provide a link to the Creative Commons licence, and indicate if changes were made. The images or other third party material in this article are included in the article's Creative Commons licence, unless indicated otherwise in a credit line to the material. If material is not included in the article's Creative Commons licence and your intended use is not permitted by statutory regulation or exceeds the permitted use, you will need to obtain permission directly from the copyright holder. To view a copy of this licence, visit http://creativecommons.org/licenses/by/4.0/ The Creative Commons Public Domain Dedication waiver (http://creativecommons.org/publicdomain/zero/1.0/) applies to the data made available in this article, unless otherwise stated in a credit line to the data. 


\section{Background}

In arthropods, external sense organs function at the interface of the environment and the organism [1-4]. Different types (and subtypes) of sense organs exist, all of which can generally be found across the arthropod body. However, some subtypes are clustered on specific appendages that are primarily used for specific behaviours, such as gustatory receptors on mouthparts or olfactory receptors on insect antennae [5-7]. External sense organs show a great variety of habitat- and behaviour-adapted forms and functions, ranging from the simple mechanosensory bristles of flies to the complex cuticular structures of crustacean feeding setae [8, 9]. This diversity raises the question of how the different shapes and functions have emerged in arthropods and which molecular mechanisms have facilitated their evolution.

Although there is no uniform classification of sense organs in arthropods, external and internal sense organs are generally distinguished from one another $[8,10,11]$. In our present study, we focus on external sense organs in insects. There are at least five morphological categories of external sense organs described across insect species: chaetoid, trichoid, basiconic, campaniform, and coeloconic sensilla $[8,10,12,13]$. This implies that they have been present in the last common ancestor of insects and that sensilla falling into the same category are therefore homologous in insects. Within the categories, sensilla can be further subdivided and assigned functions based on additional characteristics (Table 1). For example, aporous trichoid sensilla are mechanosensory organs, while multiporous trichoid sensilla function as olfactory receptors [10].

Arthropod sense organs arise from epithelial sensory organ progenitor cells (SOPs), which give rise to 4-5 different cell types (neurons, glia, sheath cells, and cells generating the cuticular structure, e.g. hair, socket) [8, 10, 14]. In Drosophila melanogaster, 5 bHLH transcription factors determine which sense organ subtypes are generated by the SOPs [10]. However, the molecular subdivision is not in line with the 5 morphological categories (Table 1) $[15,16]$; rather, it classifies the physiological function of sense organs. Members of the Achaete-Scute family (achaete (ac), scute (sc), and asense (ase)) determine external mechanosensory and gustatory sense organs, while Atonal family members (atonal (ato) and absent MD neurons and olfactory sensilla (amos)) specify (external) olfactory and internal mechanosensory organs [10, 17-20].

In the first step of sense organ development, these 5 bHLH transcription factors specify SOP fate (reviewed by [10]). In SOPs that give rise to external or internal mechanosensory organs, the SOP divides asymmetrically to give rise to two daughter cells (pIIa and pIIb), which are the precursors of the accessory (e.g. bristle, socket cell) and neural cells (e.g. sensory neuron, glial cell, sheath cell), respectively. The binary decision between these two fates is regulated by Notch $(\mathrm{N})$ and numb (nb) (reviewed by [10]). One to two additional divisions in the precursors generate all sensory cell types. At the same time as the SOP is produced, it starts to express a set of pan-neural sensory genes (e.g. snail (sna), prospero (pros), ase) [10, 21-28]. In addition, transcripts of subtype-specific genes are upregulated. The transcription factor cut (ct), for example, is expressed in external mechanosensory and gustatory sensilla, while cousin of atonal (cato) is expressed in the internal mechanosensory (chordotonal) organs and olfactory sensilla; poxneuro (poxn) and target of pox-neuro (tap) regulate chemosensory organ development [10, 29-37]. When these subtype identity genes are mutated, changes in subtype identity are observed. For example, mutations in

Table 1 Categories of external sensilla of insects

\begin{tabular}{|c|c|c|c|}
\hline Sense organ & Structure & Additional characteristics & Function \\
\hline \multirow[t]{2}{*}{ Chaetoid sensillum } & \multirow{2}{*}{$\begin{array}{l}\text { - Bristle-like } \\
\text { - Movably } \\
\text { - Articulated in wide socket }\end{array}$} & $\begin{array}{l}\text { - Uniporous (pore at tip) } \\
\text { - Steep angle }\end{array}$ & Contact chemosensory organ [16] \\
\hline & & $\begin{array}{l}\text { - Aporous } \\
\text { - Flat angle }\end{array}$ & Mechanosensory organ [15] \\
\hline \multirow[t]{3}{*}{ Trichoid sensillum } & \multirow[t]{3}{*}{$\begin{array}{l}\text { - Hair-like } \\
\text { - Articulated in tight socket }\end{array}$} & $\begin{array}{l}\text { - Uniporous (pore at tip) } \\
\text { - Steep angle }\end{array}$ & Contact chemosensory organ [16] \\
\hline & & $\begin{array}{l}\text { - Mulitporous } \\
\text { - Curved hair }\end{array}$ & Olfactory sense organ [16] \\
\hline & & - Aporous & Mechanosensory organ [16] \\
\hline Basiconic sensillum & $\begin{array}{l}\text { - Pegs } \\
\text { - Cones }\end{array}$ & $\begin{array}{l}\text { - Blunt tip } \\
\text { - Sharp or curved } \\
\text { - Flat angle when mechanosensory }\end{array}$ & Mechano- and chemosensory organ $[15,16$ \\
\hline Campaniform sensillum & - Dome with collar & $\begin{array}{l}\text { - Variations in collar size } \\
\text { - Dome shape }\end{array}$ & Proprioception \\
\hline Coeloconic sensillum & - Pit with cone inside & & Hygro/thermo-sensory \\
\hline
\end{tabular}


ct lead to transformations of external mechanosensory and gustatory sensilla into chordotonal organs [38].

Taken together, the $D$. melanogaster data show that a combinatorial code of transcription factors seems to determine sense organ subtype identity. This raises the question how this code has evolved and how, or if, it is used in other arthropod taxa or even in other insect species. Thus far, only a few studies on sense organ development in arthropods other than D. melanogaster exist. These studies have shown that genes known to be involved in sense organ subtype specification and cell type determination within the SOP lineage in D. melanogaster (ASH, ato, ase, sna, pros, Notch, Numb) are expressed during sense organ development. However, the genes seem to perform different/additional roles in different species (e.g. [39]). For example, ato shows a conserved expression in chemosensory organs of the crustacean Daphnia magna [9], the myriapod Glomeris marginata [40], and the insect $D$. melanogaster. In addition, ato is co-expressed with $A S H$ in various types of sense organs in the crustacean, including mechanosensory sensilla [9]. Does this suggest that different codes for sense organ subtype specification have evolved in insects and the closely related crustaceans? Or is the D. melanogaster code not even representative for insects? In order to address this question, we analyse here the expression and function of sensory genes in the development of different categories of sensilla in the red flour beetle Tribolium castaneum.

\section{Results}

\section{Distribution of external sensilla in the first larval stage}

In order to analyse the molecular mechanisms of sense organ development in $T$. castaneum, we first established a map of sensilla of the head and body segments of 1st instar larvae, which fell into the different morphological categories of external sensory organs (ESOs) (Table 1) and which were easily identifiable because of their prominent positions. The distribution of head sensilla has been described before [15, 41, 42], and we therefore only define here the head sensilla relevant to this study. Each antenna has one terminal trichoid olfactory sensillum (ant_TSO; Fig. 1a, b, g, k). Long mechanosensory chaetoid sensilla on the dorsal and lateral side of the head capsule are arranged in three pairs of triplets (vertex triplet (ver_tri), gena triplet (gen_tri), maxilla escort (max_esc)) on either side of the midline, and one frontal quartet (labrum quartet (lab_qua)) (Fig. 1a, b, k [41];).

In the thoracic segments, the distribution of sensilla is similar in thoracic segments 2 and 3 (t2, t3; Fig. 1a, c, k). However, thoracic segment 1 (t1) differs from $\mathrm{t} 2$ and $\mathrm{t} 3$ as it is about twice as big (Fig. 1c) and the sensilla are arranged in three anterior-posterior rows (Fig. 1c, k, anterior, medial, posterior) rather than two. Based on the morphological characteristics described in Table 1, we identified 5 chaetoid sensilla on $\mathrm{t} 1$, four of which are mechanosensory (dorsal chatoid sensilla 1 and 2 (adCSM1 and 2), anterior and posterior lateral sensilla (alCSM and $\mathrm{plCSM})$ ), while the remaining dorsal chaetoid sensillum is gustatory (pdCSG) (Fig. 1a, d, i, k). The two posterior sensilla pdCSG and plCSM are located in the same relative position in $\mathrm{t} 2$ and $\mathrm{t} 3$. In addition, $\mathrm{t} 1$ exhibits an anterior-dorsal basiconic mechanosensory sensillum (adBSM), a median transverse row of three dorsal BSMs (mdBSM1-3), and a posterior transverse row of three dorsal BSMs (pdBSM1-3; Fig. 1a, h, k). t2 and $\mathrm{t} 3$ show an anterior and posterior transverse row of BSMs only (adBSM1-4, alBSM, pdBSM1-3; Fig. 1a, d, $\mathrm{k})$. One of the anterior BSMs, alBSM, is located in a prominent position lateral to the tracheal pit in $\mathrm{t} 2$ and $\mathrm{t} 3$ and can also be identified in all abdominal segments (Fig. 1a, c, e, k). In t1, tracheal pits are absent, and the same relative position is occupied by alCSM.

Similar to $\mathrm{t} 2$ and $\mathrm{t} 3$, the sensilla are arranged in an anterior and posterior row in the abdominal segments. The anterior row consists of four BSMs (adBSM1-3 and alBSM). In the posterior row, a single BSM is visible (pdBSM; Fig. 1a, e, k). In addition, each abdominal segment has five chemosensory sensilla: two chaetoid sensilla (pdCSG1 and 2) and three trichoid sensilla (plTSO, pvTSO1 and 2). pdCSG1 and 2 are located at the dorsalposterior side of each abdominal segment and develop an open pore at the tip in the second larval stage (Fig. 1a, $\mathrm{e}, \mathrm{j}, \mathrm{k}$ ), which is typical for contact chemoreceptors. One of the three trichoid sensilla is positioned at the posterior-lateral side of the abdominal segments (plTSO), at the same vertical line and posterior to the tracheal pit (Fig. 1a, e, f, k). The remaining two trichoid sensilla (pvTSO1 and 2) are located on the ventral side of the abdominal segments. The steep insertion angle and curved shape of the TSOs suggest an olfactory function.

\section{Expression of TC ASH and TC ato in the developing sense organs}

In D. melanogaster, ac-sc and ato outline the areas where sense organs form and are therefore known as proneural genes. We analysed the expression patterns of the single T. castaneum Ac-Sc and ato homologues, Tc $A S H$ and $T c$ ato. We use here a staging system that has been developed previously for gene expression analysis in the central nervous system [43], with additional subdivisions that enabled us to capture the dynamic expression patterns of the sensory genes (Additional file 1: Figure S1) [43-47]. In the developing peripheral nervous system, clusters of $T c A S H$ and $T c$ ato positive cells are visible in the head, thoracic, and abdominal appendages as well as in domains lateral to the thoracic appendages 

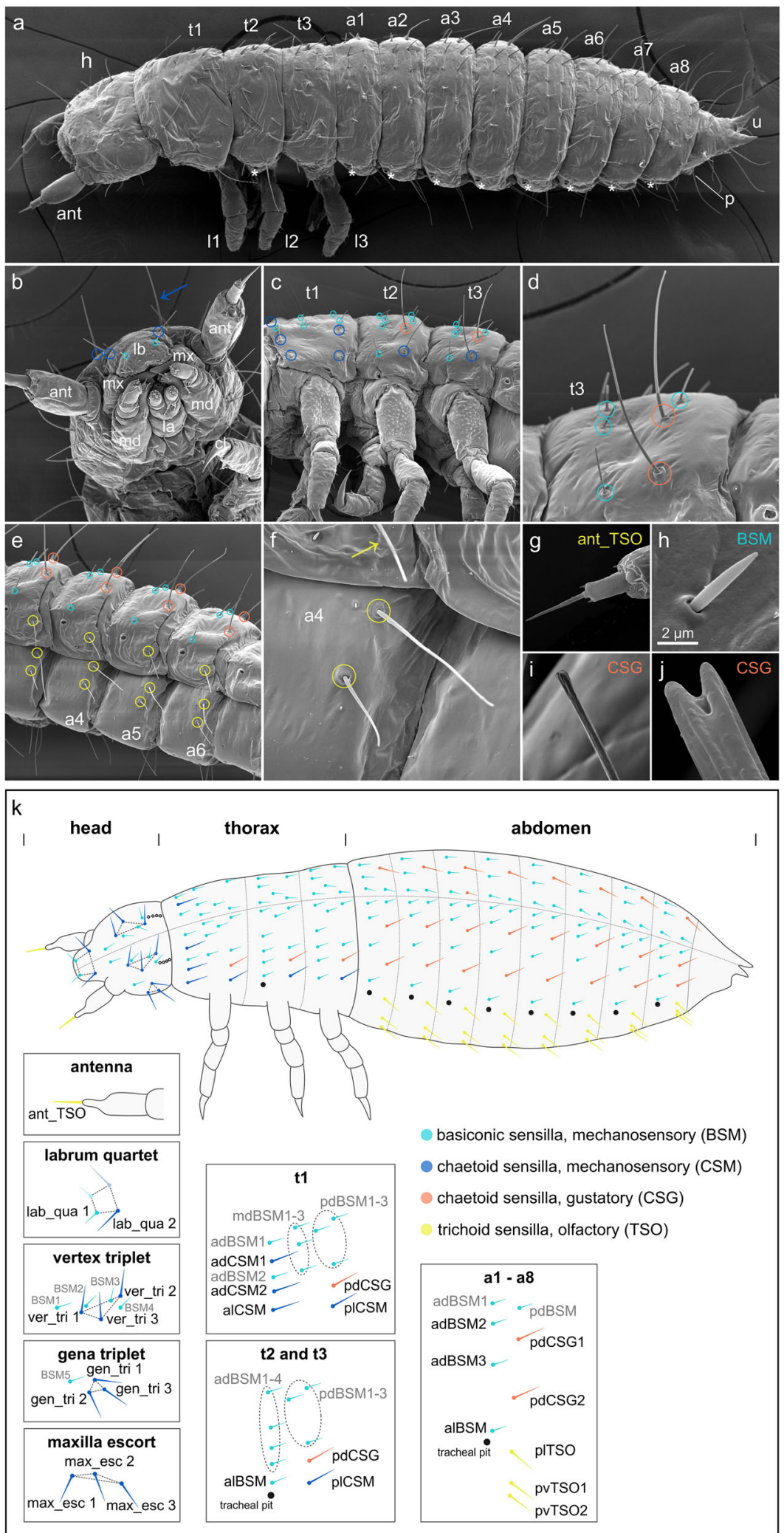

Fig. 1 (See legend on next page.) 
(See figure on previous page.)

Fig. 1 Distribution and morphology of selected external sensilla in T. castaneum larvae. Scanning electron micrographs (SEMs) of ESO morphology and distribution in the 1st larval stage (a-i), and morphology of sensilla in the 2nd larval stage (j). Anterior is towards the left in $\mathbf{a}-\mathbf{k}$. a Whole larva: asterisks, position of tracheal pits; ant, antenna; $h$, head; $t 1-t 3$, thoracic segments $1-3$; $11-13$, walking legs 1-3; a1-a8, abdominal segments 1-8; u, urogomphi; p, pygopods. b High magnification of head (ventral view) showing mouthparts: lb, labrum; mx, maxillae; md, mandibles; la, labium; and head sensilla grouped into clusters lab_qua, ver_tri, gen_tri, max_esc [41]. c, d Thoracic segments. e Abdominal segments (ventro-lateral view). $\mathbf{f}$ Abdominal TSOs, $\mathbf{g}$ ant_TSO, h BSM. i In the 1st larval stage, the CSGs have a bulb-shaped tip. $\mathbf{j}$ Open pore at the tip of a CSG at 2nd larval stage. $\mathbf{k}$ Schematic representation of 1st stage larvae showing the different types of ESOs, dorso-lateral view. Grey lettering indicates sensilla, which were not analysed in the RNAi experiments. The dashed line indicates the dorsal midline. Sensilla abbreviations, head: ant_TSO, antennal trichoid sensillum (olfactory); lab_qua, labrum quartet; ver_tri, vertex triplet; gen_tri, gena triplet; max_esc, maxilla escort. Thorax: adBSM, anterior-dorsal basiconic sensillum (mechanosensory); alBSM, anterior-lateral basiconic sensillum (mechanosensory); alCSM, anterior-lateral chaetoid sensillum (mechanosensory); dCSG, dorsal chaetoid sensillum (gustatory); dCSM1-2, dorsal chaetoid sensillum (mechanosensory) 1-2; mdBSM1-3, median-dorsal basiconic sensilla (mechanosensory) 1-3; pdBSM1-3, posterior-dorsal basiconic sensilla (mechanosensory) 1-3; plCSM, posterior-lateral chaetoid sensillum (mechanosensory). Abdomen: adBSM1-3, anterior-dorsal basiconic sensillum (mechanosensory) 1-3; alBSM, anterior-lateral basiconic sensillum (mechanosensory); dCSG1-2, dorsal chaetoid sensillum (gustatory); ITSO, lateral trichoid sensillum (olfactory); pdBSM, posterior-dorsal basiconic sensillum (mechanosensory); vTSO1-2, ventral trichoid sensilla (olfactory) 1-2

and lateral to the developing ventral nerve cord in the abdominal segments (Fig. 2a, b; Additional file 1: Figure S2). Tc ASH is also strongly expressed in the central nervous system (Fig. 2e; Additional file 1: Figure S2; see also [44]). In the areas of sense organ development, Tc ato expression starts as early as NS3, while Tc ASH expression is not visible before NS7 (Additional file 1: Figure S2). Both genes are expressed in many domains in the appendages (Fig. 2a, b; Additional file 1: Figure S2c, e, f). Here, we focus on the areas from which the sense organs depicted in the scheme in Fig. 1k arise, except for the head capsule, where gene expression cannot be related to the larval sensilla.

Tc ASH is expressed in more domains than Tc ato, in particular in regions lateral (dorsal) to the thoracic appendages and dorsal to the Tc ato domains in the abdominal appendages (bracket in Fig. 2e). In most cases, it is not possible to distinguish clusters of Tc ASH expressing cells belonging to individual sense organs in the dorsal domains because of their close proximity. Tc ASH expression in the lateral body wall starts with the areas from which alBSM and plCSM develop at NS7 to NS10 (Additional file 1: Figure S2b, c). This expression persists and the pattern develops into three rows of Tc ASH expression (Fig. 2c: anterior, medial, posterior) dorsal to the appendages in the thoracic segments covering all positions from which larval sensilla emerge (Fig. 2c; t1: pdCSM1-2, alCSM, plCSM, and all BSMs; t2 and t3: pdCSG, plCSM, and all BSMs, including alBSM). The medial row in t2 and t3 (arrows in Fig. 2c) does not exhibit external sensilla in the 1st larval stage.

Tc ato is initially only expressed in alBSM between NS7 and NS10, in addition to the appendages (Additional file 1: Figure S2e, f). At NS13.1, Tc ato is expressed in a single cluster of cells close to the posterior base of the three thoracic appendages (Fig. 2d). This position most likely corresponds to plCSM in the larva (Fig. 1k). In addition, Tc ato is expressed in a large cluster of cells in $\mathrm{t} 1$ in the same area where the tracheal pits arise in $\mathrm{t} 2$ and $\mathrm{t} 3$ (Fig. 2d). The cluster might cover the position of several sensilla (alCSM, adCSM2, adBSM2).

In the abdominal segments, the dorsal-most Tc ASH domain also spreads over the areas from which all described sensilla arise: adBSM1-3, alBSM, pdBSM, and pdCSG1-2 (Fig. 2e). Four clusters of Tc ASH positive cells are furthermore visible ventral to the dorsal domains in all abdominal segments; one is located posteriorly and in approximately the same vertical line as the tracheal pit, and three are positioned in approximately the same horizontal line posteriorly to the tracheal pit. These positions correlate with the $T c$ ato positive domains that correspond to the positions of sense organs plTSO and pdCSG2, pvTSO1 and 2 in the 1st larval stage (Fig. 2f). In addition, Tc ato is strongly expressed at the tip of the antennae, which correlates with the position of the trichoid olfactory antennal sensillum (ant TSO) in the 1st larval stage (Fig. 2b; Additional file 1: Figure S2f). Tc ASH clusters are also visible in the antenna but not at the very tip (Fig. 2a; Additional file 1: Figure S2c).

\section{Expression of genes conferring sense organ subtype identity in Drosophila: Tc ct, Tc cato, Tc tap}

In order to examine sensory organ subtype specification, we next analysed the expression of T. castaneum homologues of genes that specify subtypes in D. melanogaster. These include $T c c t, T c$ cato, and $T c$ tap. Despite numerous attempts, we were not able to obtain in situ hybridisation data for $T c$ amos, the third member of the $T$. castaneum Atonal family and poxn, for which we have functional data (see below).

Tc ct expression starts in the maxillary and labial appendages in NS7 and becomes visible in the antennae appendages by NS10 (Additional file 1: Figure S3a, b). The gene remains expressed in the labrum and whole antennae through subsequent stages (Fig. 3a, b). In 

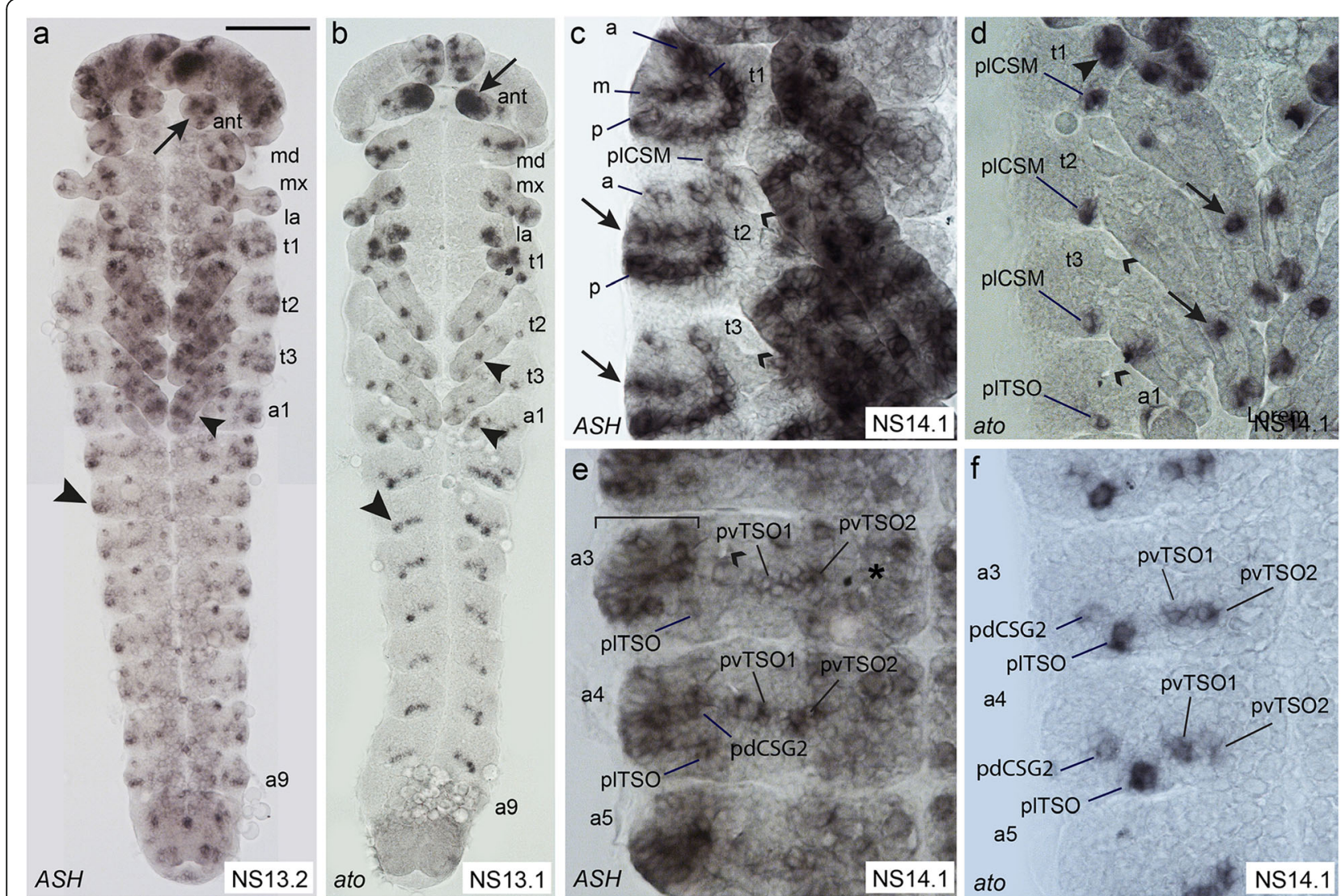

Fig. 2 Comparison of TC ASH and TC ato expression patterns in the developing peripheral nervous system. Light micrographs of flat preparations stained with DIG labelled RNA probes. Anterior is towards the top. Open arrowheads: tracheal pits. a, b Tc ASH and Tc ato expression pattern in whole embryos at NS13. Arrows: expression in the antennae. Tc ato is strongly expressed in the whole tip of the antenna, while TC ASH is expressed in small groups of cells. Small arrowheads: expression in the legs. Large arrows: expression in the lateral body wall. $\mathbf{c}$ In the three thoracic segments, TC ASH is expressed in three dorso-ventral rows (anterior-dorsal (a), medio-dorsal (m), and posterior-dorsal (p)) dorsal to the appendages, covering all areas of ESOs development. Arrows: medio-lateral expression domain in $\mathrm{t} 2$ and $\mathrm{t} 3$ corresponding to an area which is devoid of ESOs in 1st stage larvae. Transcripts are visible in the legs. $\mathbf{d}$ In the thorax, Tc ato is expressed in a single group of cells at the posterior base of the legs, corresponding to pICSM, and a few groups in the legs (arrows). At approximately the same position, the Tc ato positive plTSO cluster is visible in the abdominal segments. Arrowhead in t1: additional Tc ato positive cluster. e In the most dorsal part of the abdominal segments (bracket), TC ASH is expressed in many cells, covering the area from which the pdCSGs and BSMs arise. TC ASH is also expressed in the ventro-lateral areas from which the three TSOs arise and in the ventral neuroectoderm (asterisk). $\mathbf{f} T c$ ato is expressed in pdCSG2 and the three TSOs in the abdominal segments. For abbreviations, see Fig. 1. Scale bar in $\mathbf{a}, 100 \mu \mathrm{m}$ in $\mathbf{a}, \mathbf{b} ; 25 \mu \mathrm{m}$ in $\mathbf{c}, \mathbf{d}, \mathbf{e}, \mathbf{f}$

addition, Tc ct is strongly expressed in ring-shaped domains in $\mathrm{t} 2$ to $\mathrm{t} 3$ and a1 to a8 from NS10 onward (Fig. 3e, f, i, j, open arrowheads; Additional file 1: Figure S3b, c). These areas develop into the tracheal pits. In the thoracic and abdominal segments, low Tc ct expression becomes visible dorsal to the appendages in $\mathrm{t} 1$ to $\mathrm{t} 3$ and dorsal to the developing ventral nerve cord in the abdominal segments from stage 14.1 onward (Fig. 3e, i). At this stage, expression is also present in all appendages and the CNS (Fig. 3e, i).

In the peripheral nervous system, the fully developed Tc $c t$ expression pattern can be observed in NS14.2 (Fig. 3b, f, j). Similar to Tc ASH, Tc ct expression forms an incomplete ring-shape in $\mathrm{t} 1$, although covering a smaller region (Fig. 3f). However, in the Tc ASH positive area corresponding to the medio-dorsal row of setae in the larva (mdBSM1 to 3; Fig. 2c), only one Tc ct cluster is visible in the dorsal-most position that might correlate to expression in mdBSM1 (Fig. 3f, small arrowhead). There is no expression in the remaining part of the row, except close to the base of the first thoracic leg (Fig. 3f, large arrowhead). This area cannot be directly correlated with the position of external larval setae. In $\mathrm{t} 2$ and $\mathrm{t} 3, T c$ $c t$ expressing cells are arranged in a posterior row lateral to the appendages but appear scattered towards anterior (Fig. 3f). Similar to t1, two clusters of Tc ct positive cells are visible in the medial area in both $\mathrm{t} 2$ and $\mathrm{t} 3$; however, these cannot be correlated with external larval setae (Fig. 3f, arrows). In the abdominal segments, the fully developed $T c$ ct expression pattern covers the complete 

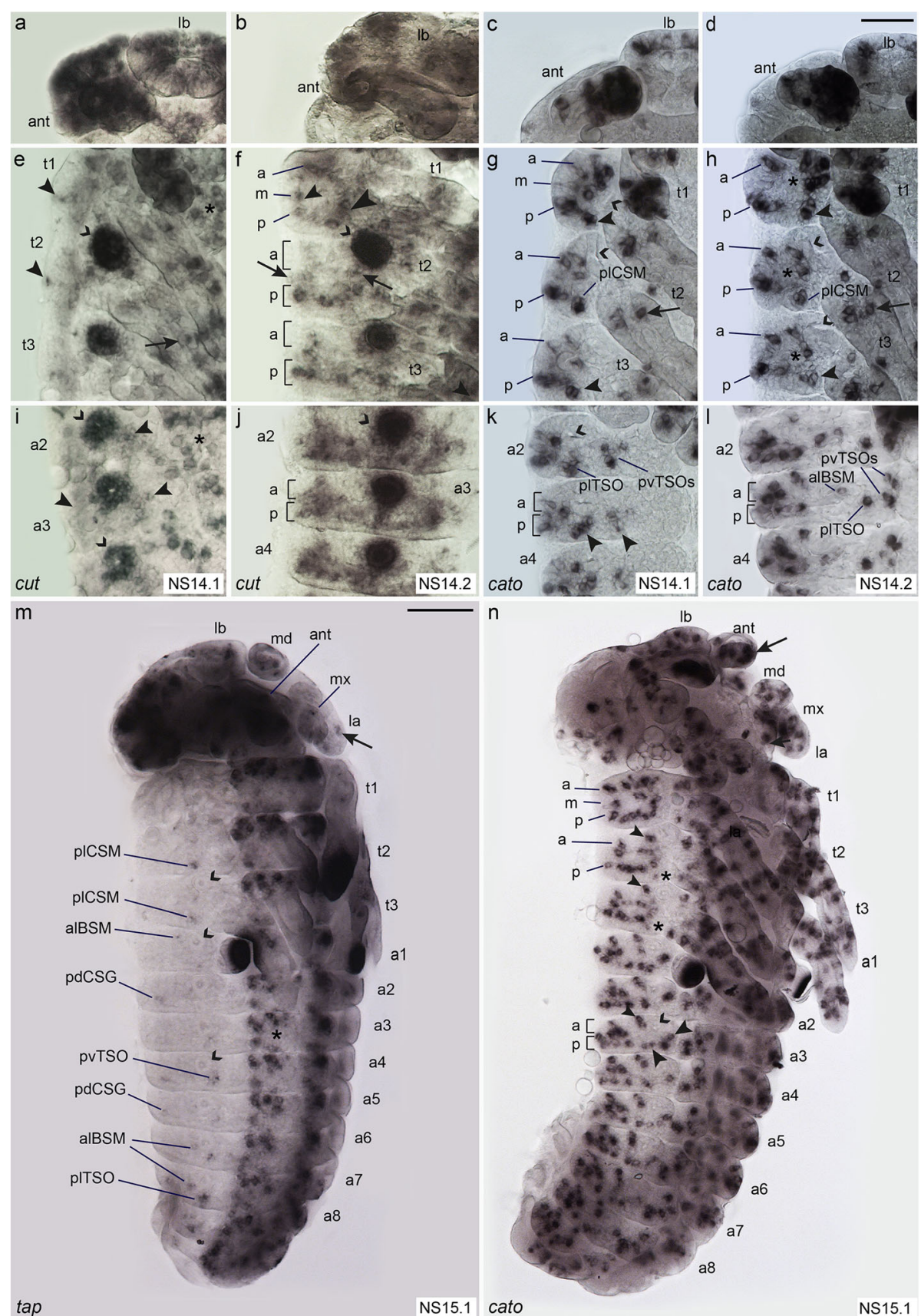

tap

NS15.1 cato

NS15.

Fig. 3 Comparison of the expression patterns of sense organ subtype-specific genes. Light micrographs of flat preparations stained with DIG labelled RNA probe of Tc ct, Tc cato, and Tc tap. Open arrowheads: tracheal pits; a, m, and p indicate the anterior-dorsal, medio-dorsal, and posterior-dorsal rows of expression, respectively, corresponding to the larval sensilla rows. a-d Tc ct and Tc cato are expressed in the labrum and antennae. e NS14.1, arrowheads: Tc ct expression lateral to the appendages. Arrow: leg expression; asterisks: VNE expression. f NS14.2, ring-like arrangement of Tc ct+ cells in t1; row $\mathrm{m}$ has two clusters, dorsal (small arrowhead) and ventral (large arrowhead). In t2-3, Tc ct+ cells are aligned in row $\mathrm{p}$ and two clusters in row $\mathrm{m}$ (arrows). Scattered, cells are visible in row a. g NS14.1: Tc cato+ clusters arranged in a, m, and p rows in t1-3. Arrowheads: plSCM clusters. Arrow: leg expression. $\mathbf{h}$ NS14.2, Tc cato is expressed in rows a, p, and a medial cluster (asterisks) in t1-3. Arrowheads: plCSM. i NS14.1-arrowheads: expression of Tc ct around abdominal tracheal pits; asterisk: VNE. $\mathbf{j}$ NS14.2: Tc ct is expressed in row p; row a is only partially covered. $\mathbf{k}$ NS14.1, Tc cato+ clusters are visible in rows a, p. pITSO and pvTSO1-2 have prominent positions posterior and ventral to the tracheal pits, respectively (arrowheads). I NS14.2: pITSO, pvTSO1-2, and alBSM indicated. $\mathbf{m}$ NS15.1, Tc tap is expressed in plCSMs, alBSMs, and pdCSGs. Arrows: expression in appendages. $\mathbf{n}$ NS15.1, clear arrangement of Tc cato positive cells in a, $\mathrm{m}$, and p rows. plCSM expression is decreased (asterisks); small arrowheads: alBSM; large arrowheads: plTSO and pvTSO1-2; arrow: expression in antenna. For abbreviations, see Fig. 1. Scale bar in $\mathbf{d}, 25 \mu \mathrm{m}$ in $\mathbf{a}-\mathbf{l}$; scale bar in $\mathbf{m}, 100 \mu \mathrm{m}$ in $\mathbf{m}, \mathbf{n}$ 
posterior row of larval setae, including the lateral and ventral TSOs medial to the tracheal pits (Fig. 3j). The anterior and posterior expression domains form a continuous area, which takes on a triangular shape towards anterior and thus does not cover the positions of all larval setae in the anterior row (Fig. 3j).

In the peripheral nervous system, Tc cato is first expressed in the antennae and mandibles in stage NS7 embryos (Additional file 1: Figure S3d). In subsequent stages, additional Tc cato expression sites form in the antennal, maxillary, labial, and thoracic appendages (Fig. 3c, g; Additional file 1: Figure S3f). From stage NS10 onward, clusters of Tc cato expressing cells become visible lateral to the appendages in the thorax and lateral to the ventral neuroectoderm in the abdominal segments (Additional file 1: Figure S3f). With the appearance of additional expression domains, the clusters become arranged into rows (Fig. 3g, h, k, l, n). The developing plCSM, alBSM, plTSO, and pvTSO1 and 2 sensilla are clearly visible as separate clusters, while the remaining clusters merge into each other and are not identifiable relative to the position of the larval sensilla (Fig. 3g, h, k, l, $\mathrm{n})$. The arrangement of Tc cato positive cells into rows is more pronounced in stage NS15.1, possibly due to the segments having narrowed along the anterior-posterior axis and extended along the dorsoventral axis as part of germband retraction and dorsal closure (Fig. 3n). The posterior rows of Tc cato expression in the thoracic and abdominal segments seem to cover all larval sensilla positions, except for plCSM, where the previous expression has almost ceased. Three clusters in the medial row of $\mathrm{t} 1 \mathrm{might}$ correspond to the medio-dorsal BSMs1-3 (Fig. 3n). Similar to Tc ASH expression, a medial row of expression is also visible in $\mathrm{t} 2$ and $\mathrm{t} 3$, which cannot be correlated to external larval sensilla (Fig. 3n). In t2, $\mathrm{t} 3$, and the abdominal segments, there is a gap of $T c$ cato expression in the medial part of the anterior rows. In abdominal segments, however, several $T c$ cato positive cell groups cluster in the dorsal part of the anterior row, which might rearrange to cover the gap during further dorso-ventral extension of the germband (Fig. 3n). Furthermore, many Tc cato clusters are visible in all appendages and there is a strong expression domain at the tip of the antennae (Fig. 3n).

Tc tap is strongly expressed in the CNS from NS11 onward (Additional file 1: Figure S3e); however, expression is comparably low in the peripheral nervous system. It does not start before NS15.1 (Fig. 3m) and begins to decrease after about $6 \mathrm{~h}$ in NS15.4 (Additional file 1: Figure S3g). Tc tap is expressed in clusters of cells in the head appendages and in a few cells in the lateral body wall of the thorax and the abdomen (Fig. 3m). Tc tap positive cells are identifiable in the plCSMs, alBSMs, plTSOs, and pvTSOs as well as in one of the two abdominal pdCSGs (Fig. 3m; Additional file 1: Figure S3g).

\section{Expression patterns of Tc asense, Tc prospero, and Tc snail} In D. melanogaster ase, pros and sna are so-called panneural genes, which are expressed in all sense organs after SOP formation. Tc ase shows a strong and prolonged expression in the CNS (Fig. 4a [43, 44];); however, the expression is limited to a transient expression in a few cells and clusters in the PNS (Fig. 4a). Expression in the PNS starts at NS9 in small domains on the appendages and a few cells on each side of the lateral body wall (Additional file 1: Figure S4a). At NS11, additional clusters appear in the abdominal segments (Additional file 1: Figure S4b). One of the clusters can be identified as plTSO after formation of the tracheal pits in the abdominal segments at NS13 (Fig. 4a). The expression domain located dorsal and posterior to the tracheal pits cannot be assigned to specific sensilla (Fig. 4a). Tc ase expression decreases thereafter and is not detectable any more in most areas of the PNS by NS15 (Additional file 1: Figure S4f).

Tc pros expression is first visible in the head appendages at NS7 (Additional file 1: Figure S4c). At NS10, a single cluster of Tc pros positive cells appears on each side of the lateral body wall (Additional file 1: Figure S4g). By NS13.4, additional clusters are present, three of which can be attributed to the developing plCSMs (thorax) and plTSOs (abdomen) and the alBSMs (Fig. 4b). At NS15.1, Tc pros positive clusters seem to cover all areas of external sensilla formation (Fig. 4d). In addition, Tc pros is expressed in the medial area in $\mathrm{t} 2$ and $\mathrm{t} 3$ that does not give rise to external sensilla (Fig. 4d). Due to their prominent positions around the tracheal pits, alBSM, plCSM, plTSO, and pvTSO1 and 2 can be clearly identified (Fig. 4d).

Tc sna expression starts at NS7 in the mandibles, and by NS9, all appendages show Tc sna expression domains (Additional file 1: Figure S4d). Similar to Tc pros, bilateral Tc snail positive clusters appear in the lateral body wall. They appear first in the thoracic segments at NS7 and have extended to A9 by NS9 (Additional file 1: Figure S4d, e). One additional Tc sna expression domain is visible at NS13, which can be allocated to the abdominal plTSO due to its position posterior to the tracheal pits (Fig. 4c). During the subsequent stages, Tc sna shows a transient expression pattern in groups and single cells, some of which cover the areas where sensilla appear next to landmarks, such as the plCSMs in the thoracic segments (Fig. 4e). Tc sna expression decreases earlier than that of Tc pros in the PNS, although both 


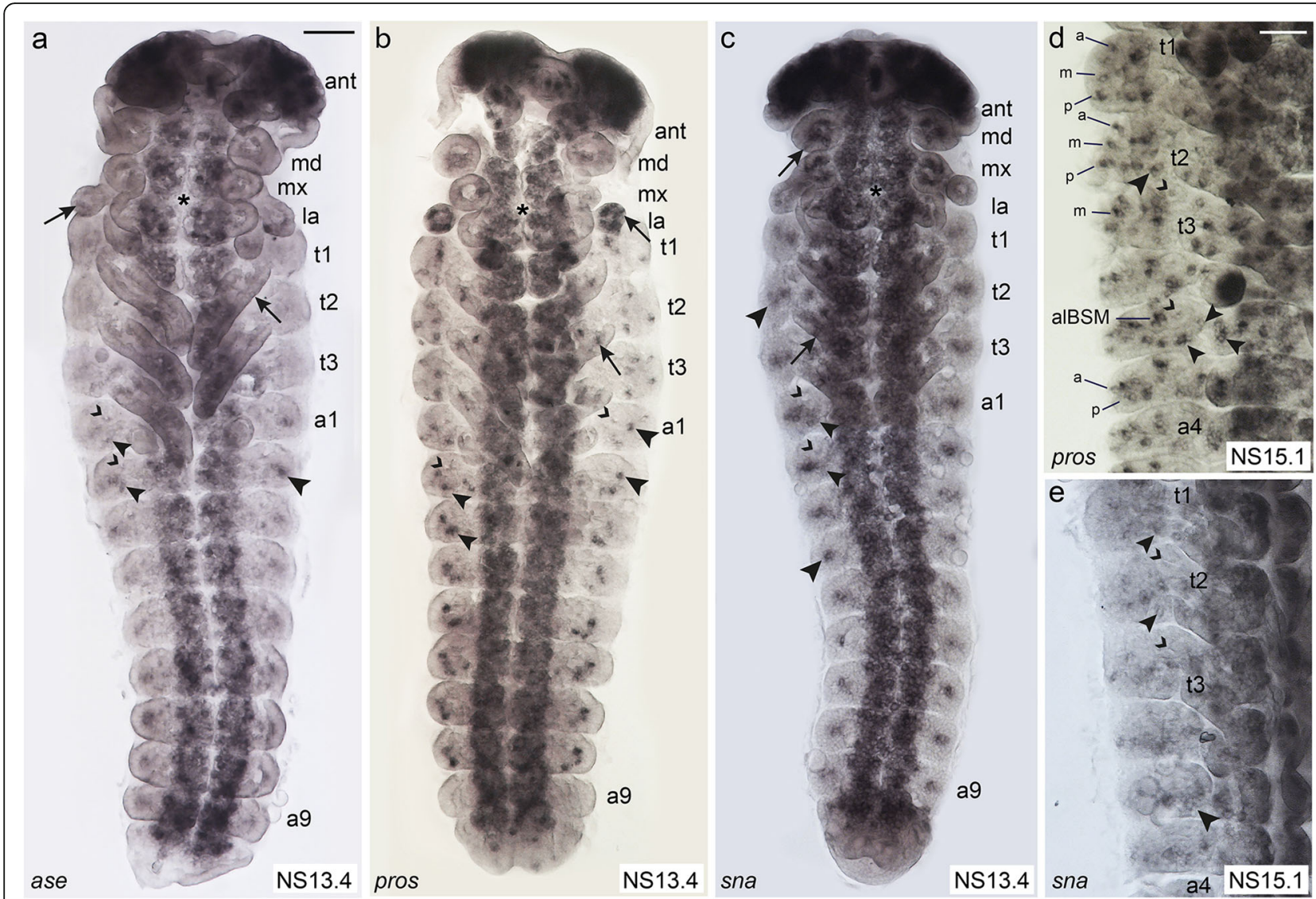

Fig. 4 Comparison of the expression patterns of pan-neural genes. Light micrographs of flat preparations stained with DIG labelled RNA probe of Tc ase, Tc pros, and TC sna. Open arrowheads: tracheal pits. a TC ase is strongly expressed in the developing brain, the antennae, and the VNE (asterisk). Arrows: scattered Tc ase+ cells in the remaining appendages (arrows) and the lateral body wall (large arrowhead). Small arrowheads: pITSOs. b Small arrowheads: Tc pros expression in pITSOs. Large arrowheads: Tc pros expression dorsal to the tracheal pits in alBSMs. Tc pros is also strongly expressed in the developing brain, in clusters of cells in all appendages (arrows) and the VNE (asterisk). c Similarly, Tc sna is expressed in the brain, clusters of cells in the appendages (arrows) and VNE (asterisk). Large arrowheads: Tc sna is expressed in large clusters in the lateral body wall. The expression extending below the tracheal pits might correspond to the developing plTSOs (small arrowheads). $\mathbf{d}$ At NS15.1, groups and single cells express Tc pros in the lateral body wall, which seem to cover all areas of ESO formation. Additionally, the medial row that does not give rise to ESOs expresses TC pros. Due to their prominent positions relative to the tracheal pits, the alBSM clusters, the plCSM clusters (large arrowhead), and the three TSO clusters (small arrowheads; pITSO, pvTSO1-2) are easily identifiable. e Tc sna shows a transient expression pattern in groups and single cells, some of which cover the areas where sensilla appear next to landmarks, such as the plCSMs (small arrowheads) in the thoracic segments and the pITSOs in the abdominal segments (large arrowhead). For abbreviations, see Fig. 1. Scale bar in a, $50 \mu \mathrm{m}$ in $\mathbf{a}-\mathbf{c}$; scale bar in $\mathbf{d}, 50 \mu \mathrm{m}$ in $\mathbf{d}$, e

genes continue to be strongly expressed in the CNS (Fig. 4d, e).

\section{Tc ASH and Tc ato have different roles in sense organ development}

The different expression of Tc ASH and Tc ato in SOPs (Fig. 2) raises the possibility that these genes specify sense organ subtypes like in D. melanogaster. In order to test this hypothesis, we disrupted Tc ASH and Tc ato function via parental RNAi and examined sense organs in affected larvae. We focused our analysis on a subset of sensilla which were easily identifiable because of their proximity to landmarks (tracheal pits, segmental border) or arrangement relative to other sensilla. The grey lettering in Fig. 1k indicates which sensilla were not included in the RNAi study.

When examining control larvae (from parents injected with water or buffer alone), 98 to $100 \%$ of these sensilla were present (Figs. 5a and 6a; Additional file 1: Table $\mathrm{S} 1)$. The observed variation is due to the absence of sensilla at specific positions along the anterior-posterior axis. In the head and thorax, $99.1 \%$ of the analysed sensilla are present at all positions $(2461 / 2484)$, while in the abdominal segments overall 2.87\% (199/6912) of sensilla are missing. The abdominal TSOs (4.32\%) show the highest variability followed by the CSGs $(2.60 \%)$. In order to elucidate the significance of the RNAi phenotypes, we recorded and analysed the affected sensilla 


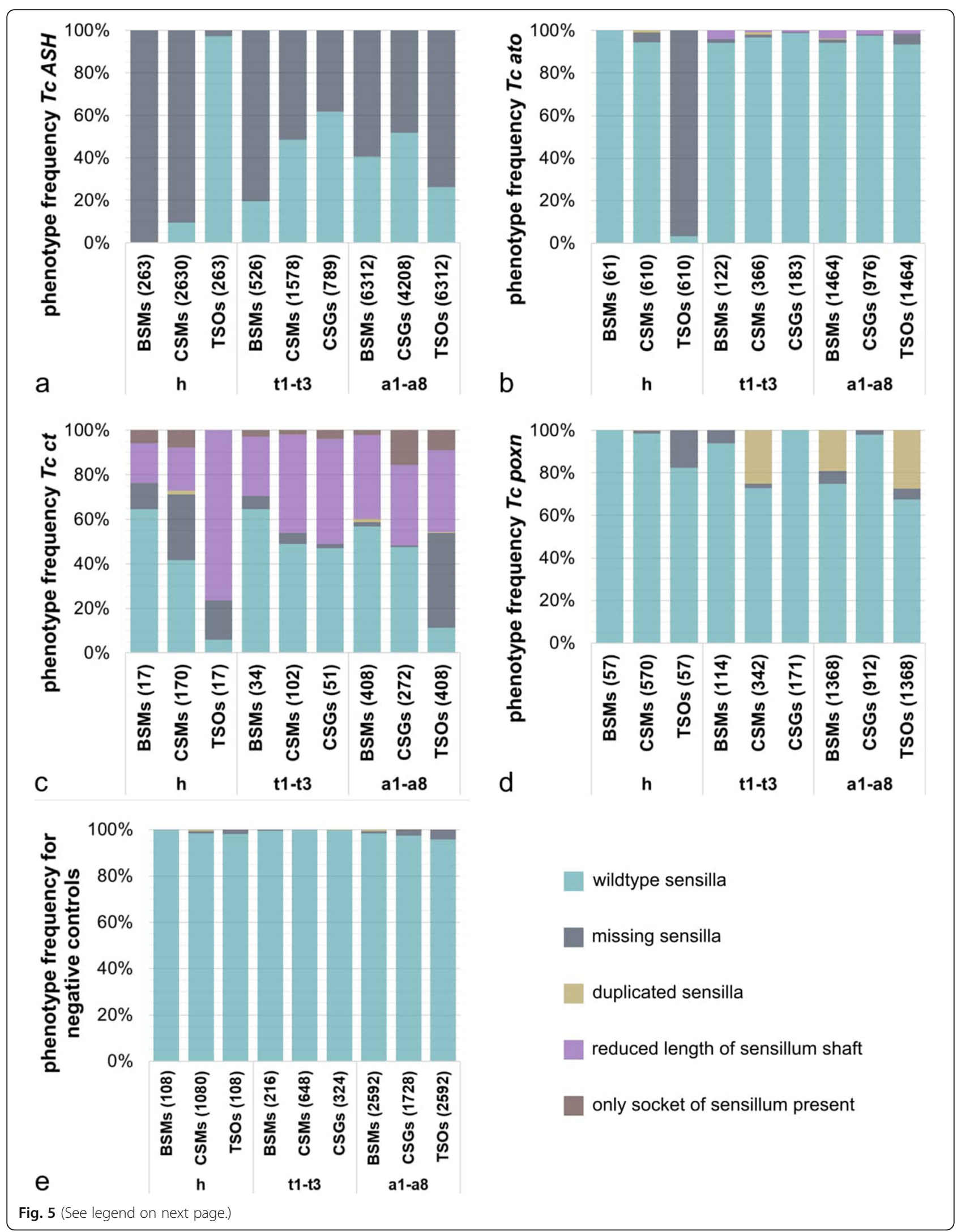


(See figure on previous page.)

Fig. 5 Quantification of the RNAi phenotypes of external larval sensilla. The bars represent the percentages of phenotypes identified for the different ESO subtypes (BSMs, CSMs, CSGs, and TSOs) on the head (h), the thoracic (t1-t3), and the abdominal segments (a1-a8) of Tc ASH (a), Tc ato (b), Tc ct (c), Tc poxn RNAi (d), and the negative control cuticles (e), respectively. Sensilla that were not affected are categorised as 'wildtype sensilla'. Sensilla showing a phenotype are divided into the four categories 'missing sensilla', 'duplicated sensilla', 'reduced length of sensillum shaft', and 'only socket of sensillum present', if applicable. a We analysed 387 specimens for both non-overlapping dsRNA fragments (NOF1 and 2) of TC ASH larvae in total, of which 263 showed a specific phenotype (sensilla missing). b We analysed 119 specimens of TC ato RNAi (NOF1 and NOF2 collectively), of which 61 showed a phenotype. Tc ato RNAi cuticles were missing the ant_TSOs (96.72\%, see 3rd bar), and also observed a small percentage of duplicated sensilla and sensilla with reduced shaft lengths. c We were able to analyse only 26 specimens in total for both NOFs of Tc ct ( $n=17$ showed a phenotype). Sensilla of Tc ct cuticles could be grouped into the four different categories of phenotypes. The most abundant phenotype for all ESOs types was identified as 'sensilla with reduced shaft lengths' (purple). $\mathbf{d}$ We performed pRNAi in T. castaneum pupae to examine the function of Tc poxn. We analysed 111 specimens in total. 51.35\% of the analysed specimens showed a phenotype which were identified as duplicated sensilla (CSMs on thorax, BSMs and TSOs on abdomen). See Additional file 1: Table S4 for summary of RNAi injection results

separately for the head, thorax, and abdomen (Fig. 5; Additional file 1: Figure S5). For Fig. 5, we put the numbers of affected sensilla together for each sensilla category (TSOs, CSMs, BSMs, CSGs) across all larvae analysed (see Additional file 1: Tables S2-6 for summary of RNAi data).

In Tc ASH RNAi larvae, two types of morphologically distinct mechanosensory sensilla (basiconic and chaetoid) are missing from the head: $90.38 \%$ of the CSMs and $99.62 \%$ of the BSMs (Figs. $5 \mathrm{a}$ and $6 \mathrm{~b}-\mathrm{d}$ ). In addition, $2.73 \%$ of the olfactory antennal trichoid sensillum ant TSO are missing; however, this is only slightly above the proportion of defects seen in control larvae (1.85\%; Fig. 5d; Additional file 1: Table S1). A similar pattern is seen on the thorax, where BSMs are most affected (80.42\% missing) and to a lesser extent the CSMs (51.52\% missing) (Fig. 6c) In addition, 38.15\% of the gustatory chaetoid sensilla, the CSGs, are absent on the thorax (Fig. 5a). The intermediate phenotype in the CSMs could indicate that Tc ato, which is expressed in the thoracic CSMs, can partially replace the role of $T c$ $A S H$. Alternatively, other proneural and/or positional factors act together with ASH, which have not been analysed here. This might also explain the low effect of $T c$ $A S H$ RNAi on the CSGs which do not co-express $T c$ ato.

Although the head TSO pattern is not significantly changed in Tc ASH RNAi larvae, the TSOs are the most affected sensilla in the abdominal segments. $73.80 \%$ of the TSOs (plTSOs, pvTSOs 1 and 2) are missing in the abdomen, followed by $59.28 \%$ of the BSMs and $48.05 \%$ of the CSGs (Figs. 5a and 6d). In the most severe $T c$ ASH RNAi phenotypes, all sensilla are missing (Additional file 1: Figure S5d, $\mathrm{f}, \mathrm{j}$ ). This is in line with previous Tc ASH RNAi data in adult beetles [48]. In addition to the sensilla phenotypes, the pretarsal segments of the legs, the urogomphi, and the mandibles appear rounded (Additional file 1: Figure S5b, h, l compare to Additional file 1: Figure S5a, g, k). The 'rounded pretarsal segment' phenotype has also been documented in the iBeetle
RNA interference screen and in another insect $[49,50]$. Furthermore, the tarsal claws are missing in Tc ASH RNAi adult beetles [48]. Taken together, our results clearly show that $T c A S H$ is required for the generation of all subtypes of ESOs analysed, except for the antennal TSOs.

In contrast to $T c A S H, T c$ ato has only a minor role in the formation of larval ESOs. This is similar to D. melanogaster larvae, where ato is only required in a single combined olfactory/gustatory dorsal organ besides regulating the development of internal stretch receptors [17, 51]. In T. castaneum, the antennal TSOs are the only ESOs that are frequently missing (96.72\%) in Tc ato RNAi (Fig. 5b, dark blue bar; Fig. 6e). All other ESOs are present on the head, and on the thorax and abdomen, BSMs, CSMs, CSGs, and TSOs are only missing to a small percentage, which is in the range of the variations seen in the controls (0.55-4.92\%; Fig. 5b). The differentiation of all types of sensilla is also affected at a small rate in the thorax and abdomen: the sensilla are duplicated or have shorter shafts compared to wildtype (0.1-1.37\%, beige and $0.55-4.10 \%$, purple in Fig. $5 \mathrm{~b}$ ). While this could be due to off-target/toxic effects of the dsRNA, Tc ato could also function during sensilla morphogenesis at a late stage of development that was not captured in our analysis.

\section{Functional analysis of the 'subtype' identity genes}

Next, we analysed the role of the T. castaneum homologues of genes that specify subtype identity in $D$. melanogaster, namely $T c c t, T c$ poxn, Tc amos, Tc cato, and Tc tap. Parental Tc ct RNAi resulted in sterile females, and we therefore performed embryonic RNAi to examine gene function. In $T c$ ct RNAi larvae, all types of ESOs are affected (Figs. $5 \mathrm{c}$ and $6 \mathrm{~h}-\mathrm{j}$ ). However, compared to the Tc ASH phenotype, most of the sensilla are present in Tc ct RNAi larvae but they show differentiation defects. We categorised these defects into 'duplicated sensilla', 'reduced length of sensilla shaft', 'only socket of sensilla present', 'sensilla missing', and 'wildtype' (Fig. 5c). The TSOs are most strongly affected in 


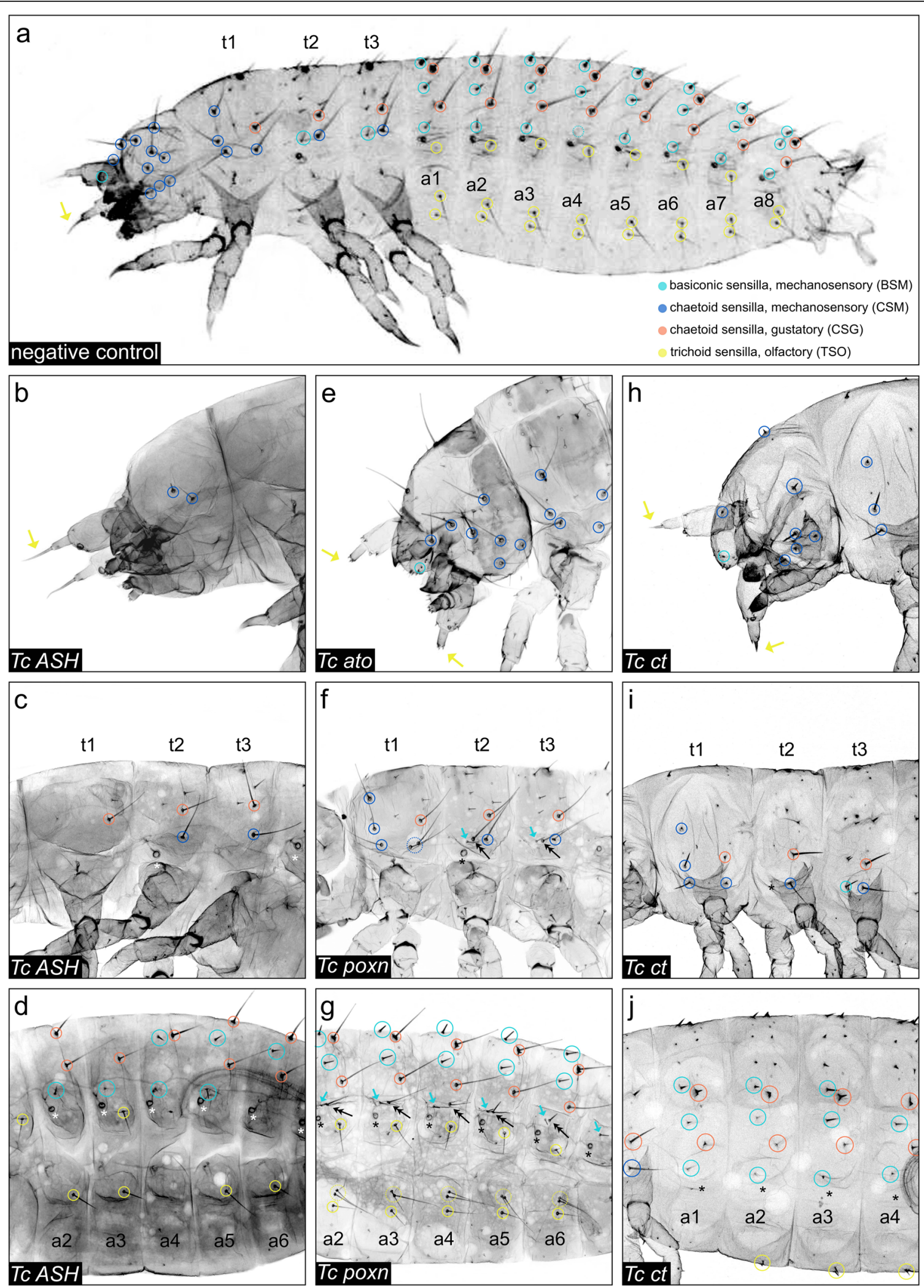

Fig. 6 (See legend on next page.) 


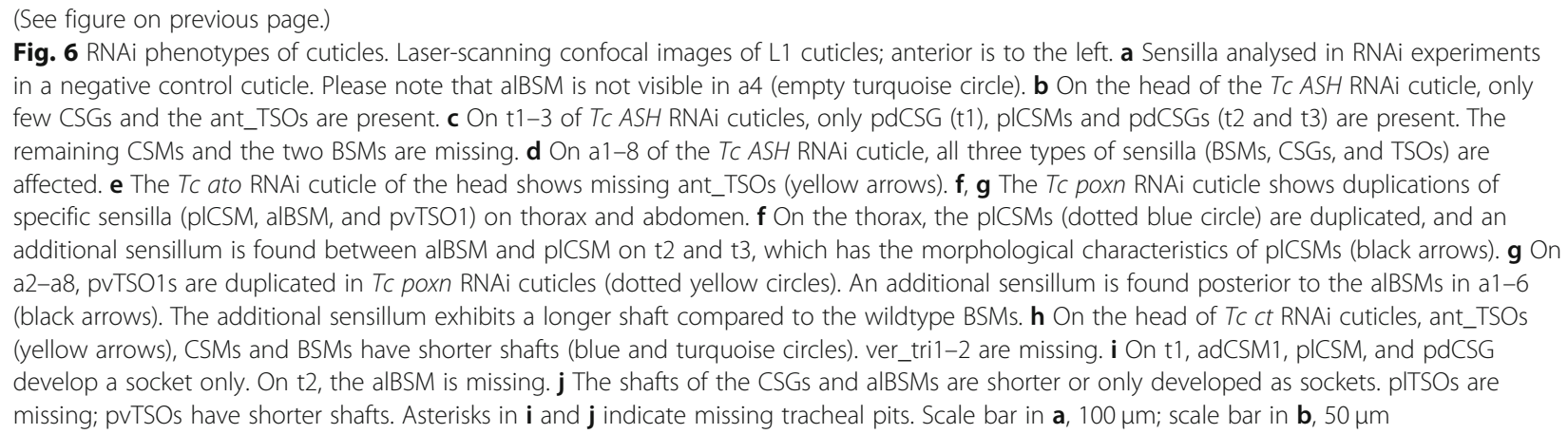

Tc ct RNAi. On the head, the ant_TSOs are affected in $94.12 \%$ of the cases, while $89.22 \%$ of the abdominal TSOs that show abnormal shape (Fig. 5c, turquoise). The predominant phenotype for all TSOs is a reduction in sensilla lengths $(76.47 \%$ of ant_TSOs and $36.76 \%$ of abdominal TSOs; Fig. 6h, j, yellow arrows and circles).

The CSMs are the second most affected ESO subtype in Tc ct RNAi larvae: $58.24 \%$ show a phenotype in the head and $50.98 \%$ in the thorax (Fig. 6h, i; blue circles). There is a difference in the distribution of the sensilla phenotypes between head and thorax. In more than half of the affected CSM sensilla positions in the head, sensilla are absent (29.41\%), while reduced length of sensilla shaft is the predominant phenotype in the thoracic CSMs (44.12\%, Fig. 5c). Furthermore, $52.57-52.94 \%$ of the thoracic and abdominal CSGs are affected (Fig. 6i). The most prominent phenotype is again the reduced length of the sensilla shaft (36.40-47.06\%). However, the abdominal CSGs show the highest percentage of the 'only socket of sensilla present' phenotype (15.44\%) compared to all other sensilla types (Fig. 5c, brown bars). The BSMs are about equally affected across all body parts $(35.29 \%$ in the head and thorax, $43.14 \%$ in the abdomen; Figs. 5c and 6i, j). Again, there are slight differences in the distribution of the sensilla phenotypes. In the head, about one third of the BSMs are missing in the affected positions, while differentiation defects are predominant in the affected BSMs of the remaining body parts (Fig. 5c). In addition, tracheal pits are absent in $96.15 \%$ of the Tc $c t$ RNAi larvae, which corresponds with the prominent circular expression of the gene in the areas where the tracheal pits develop (Fig. 3e, f, i, j, open arrowheads; Additional file 1: Figure S3b, c; Fig. 6i, j; black asterisks indicating missing tracheal pits) and is in line with a previous Tc ct RNAi study [52]. Ct function in tracheal development seems to be conserved in insects $[53,54]$. Taken together, Tc $c t$ is required to various degrees for the development of all ESO subtypes. The position-specific Tc $c t$ RNAi phenotype suggests that local cues influence the differentiation of the sensilla either, for example, by modulating $T c$ ct expression or by providing additional differentiation factors that regulate sensilla morphogenesis.

In contrast to the Tc $c t$ RNAi phenotype, only a small subset of sensilla is affected in Tc poxn RNAi larvae. In $\mathrm{t} 1$ to $\mathrm{t} 3$, the mechanosensory sensillum plCSM is duplicated $(50.88 \%)$, and in the abdominal segments, the mechanosensory alBSM (55.92\%) and the olfactory pvTSO1 (81.80\%) show the same duplication phenotype (beige bars in Fig. 5d; double arrowhead in Fig. 6f, g; yellow dotted circles in Fig. 6g).

We also attempted to analyse a gene that is regulated by Poxn in D. melanogaster, target of pox neuro (tap) [30]; however, Tc tap RNAi larvae were not analysable because of gross morphological defects such as the absence of the abdomen or deformation of head and thorax. These results are in line with those of the iBeetle screen [49]. We also attempted to functionally analyse two additional members of the Atonal family, Tc amos and Tc cato. However, similar to Tc tap RNAi, severe structural defects made it impossible to analyse the sensilla pattern in Tc cato RNAi larvae.

\section{Discussion}

We show here that the expression and role of the proneural genes Tc ASH and Tc ato and homologues of the $D$. melanogaster subtype identity genes are not limited to specific categories of sensilla. This suggests that, in contrast to $D$. melanogaster, the concept that the identity of categories of sense organs can be assigned to the function of single or combinations of transcription factors cannot be applied in T. castaneum. Regardless of how we categorise the sense organs-by morphology, by function, or both-there is no transcription factor code that can be aligned with a single category. In the following, we discuss how the functions of the sensory genes have diverged in homologous classes of $D$. melanogaster and $T$. castaneum sensilla and how these findings might 
contribute to our understanding of the evolution of sense organ diversity in arthropods.

\section{Proneural gene expression and sense organ subtype identity are not directly linked in $T$. castaneum}

A striking discovery of our study is that in T. castaneum, a single proneural transcription factor, Tc $A S H$, is required for the formation of all morphological and functional classes of ESOs analysed. This includes olfactory trichoid sensilla (TSOs), gustatory chaetoid sensilla (CSGs), and two types of mechanosensory sensilla, chaetoid (CSMs) and basiconic (BSMs). In contrast, in D. melanogaster, available molecular data on adult sense organs on the thorax and wing show that Ac and Sc determine (largely redundantly) the identity of external mechanosensory (trichoid and chaetoid) and gustatory sensilla (chaetoid), excluding olfactory sense organs [55-59].

Similarly, the role of the proneural gene Ato has diverged in D. melanogaster and T. castaneum. In D. melanogaster, Ato is essential for the development of a subset of adult olfactory sensilla on the head, the coeloconic sensilla on the antennae, and the basiconic sensilla on the maxillary palps [60] but it is not required in the olfactory trichoid sensilla which are specified by amos [34]. In contrast, in T. castaneum, Tc ato is expressed in trichoid (ant_TSO, abdominal TSOs), basiconic (alBSM), and chaetoid sensilla (alCSM, plCSM, pdCSG2). However, Tc ato is only required for the formation of one of these sense organs, the ant_TSOs $(96.72 \%$ ant_TSOs absent in Tc ato RNAi), while a specific external morphological phenotype was not observed in the remaining ESOs. This includes the abdominal TSOs, which coexpress Tc ASH and are morphologically different from the ant_TSOs (longer and thinner), suggesting they might represent a subcategory of trichoid olfactory sensilla in the T. castaneum larva. Future analysis will show if Tc ato plays a role in the differentiation of the internal TSO cell types (e.g. neuron, sheath cell), which we have not analysed here. Alternatively, it acts redundantly to Tc ASH as a proneural gene to increase the robustness of the system, similar as $a c$ in $D$. melanogaster $s c$ positive SOPs [58]. The same could also apply for the remaining Tc ato/Tc ASH positive sensilla (plCSM, pdCSG2) that do not show a phenotype in Tc ato RNAi. Vice versa, Tc ASH could have a redundant role or could be involved in the differentiation of ant_TSO, where it is co-expressed with Tc ato and does not show an RNAi phenotype. In this context, it is interesting to note that in the D. melanogaster larva, which does not exhibit antennae, Ato and Sc are required in a unique composite olfactory/gustatory dorsal organ on the head [51]. Sc seems to control the development of the non-olfactory sensory neurons, which are absent in the sc mutant larva.
The requirement of Ato in antennal sense organs seems to be conserved in Mandibulata (insects, crustaceans, myriapods), since Ato is expressed during the development of diverse prominent olfactory sense organs at the distal antennal tip of two other arthropod taxa, the aesthetascs of crustaceans on the 1st pair of antennae and the cone sensilla of millipedes $[9,40]$. This suggests that Ato was recruited for the formation of antennal olfactory sense organs at the same time as antennae evolved in the last common ancestor of the Mandibulata and that these sense organs then diverged to adapt to different habitats.

\section{The regulation of subtype identity genes has diverged in T. castaneum}

In D. melanogaster, the determination of sense organ subtype identity seems to be a decision between two (or three) alternative developmental pathways. Misexpression and loss-of-function studies show that Ac-Sc promote external mechanosensory versus internal mechanosensory (chordotonal organ) fate, while Ato supports formation of olfactory versus gustatory/ mechanosensory sensilla externally, in addition to internal mechanosensory organ versus external mechanosensory organ fate $[17,23,24,32,61,62]$. Subtype identity genes acting downstream of the proneural genes such as $c t$ and poxn play a central role in determining the alternative fates [38, 63-65].

We show here for the first time that D. melanogaster subtype identity genes are involved in sense organ development outside the dipterans suggesting that these genes belonged to the sense organ toolkit at least in the last common ancestor of insects if not beyond. We tested five $D$. melanogaster homologues for potential roles in subtype identity: $c t$, poxn, the neurogeninrelated gene tap, and two additional members of the Ato family, amos and cato. However, we did not find a specific role of the analysable genes (Tc ct, Tc poxn) in subtype identity in $T$. castaneum since we neither observed a transformation of one sensilla type into another, nor loss or differentiation defects in specific morphological or functional sensilla classes.

Tc $c t$ is expressed in all types of analysed sensilla, and the predominant phenotypes in Tc ct RNAi larvae are differentiation defects, such as shorter sensilla and missing sensilla shafts ('sockets only', Fig. 5). In D. melanogaster ct mutants, however, external larval and adult mechanosensory organs are transformed into chordotonal organs [38, 63, 65]. Furthermore, the observation that $T c$ ct is expressed in all sensilla types, including those that (co-)express Tc ato, implies that the sensory gene network has diverged in insects since $D$. melanogaster Ato executes its subtype identity by suppressing $c t$ expression [32]. A co-requirement of Tc ato and Tc ct in 
sense organ development is supported by the fact that in Tc ct RNAi larvae, the antennal olfactory sense organ ant_TSO is affected to a similar degree as in Tc ato RNAi larvae (Tc ct RNAi, 94\%; Tc ato RNAi, 97\%). However, the predominant phenotype in Tc ct RNAi larvae is a reduction in sensilla lengths, rather than the loss of the sensilla, indicating that Tc ct is involved in the differentiation of ant_TSO. Furthermore, we observed a Tc ct RNAi phenotype in the remaining TSOs that co-express Tc ato, Tc ct, and Tc ASH; the abdominal plTSO; and pvTSO1 and 2. Although not substantially affected in $T c$ ato RNAi larvae, these olfactory sensilla show a high percentage of differentiation defects in Tc $c t$ RNAi larvae (89\%) and are frequently absent in Tc ASH RNAi larvae (74\%). To conclude, in T. castaneum, ct does not confer subtype identity; rather, the gene product seems to play a role in sensilla morphogenesis in all sense organs.

Similarly, the requirement of Tc poxn is not restricted to a specific class of sensilla; however, like in D. melanogaster, the gene is only needed in a small subset of larval sensilla [66]. In Tc poxn RNAi larvae, all functional types of the analysed sensilla are duplicated: gustatory (plCSM), mechanosensory (alBSM), and olfactory (pvTSO1). This is in contrast to D. melanogaster, where Poxn is required in the class of polyinnervated gustatory sensilla in larvae and adults [66, 67]. In larvae, for example, three types of polyinnervated sensilla (two basiconic sensilla ('kölbchen') per thoracic hemi-segment; one campaniform sensillum ('papilla') and one trichoid sensillum ('hair') per abdominal hemi-segment) are transformed into monoinnervated mechanosensory organs in poxn mutants $[66,68]$. The observed duplication in Tc poxn RNAi larvae might be either due to a duplication of the SOPs or within the SOP lineage. The latter is supported by a detailed study of the sensilla lineages in D. melanogaster poxn mutant larvae showing that Poxn is required in the progeny of the SOPs and regulates the number and types of cells produced by each secondary precursor [66]. The three sensilla types affected in Tc poxn RNAi larvae (plCSM, alBSM, and pvTSO) express Tc tap suggesting that Tc tap might be activated by Tc poxn and expressed in the same sensilla as it is the case in D. melanogaster larvae [30]. Functional studies of Tc tap could have contributed to our understanding of the regulatory mechanisms in poxn positive sense organs, but unfortunately, the Tc tap RNAi larvae were not analysable. Taken together, we found no obvious role of Tc poxn in subtype identity; rather, the duplication phenotype suggests a role in sensory organ cell lineage regulation.

We attempted to analyse the function of the Atonal family genes $T c$ amos and Tc cato, but severe overall morphological defects prevented us from studying the sensilla pattern. Based on our gene expression analysis, however, Tc cato does not show sense organ subtype-specific expression: it is expressed in all morphological and functional types of sensilla. Expression starts after formation of the SOPs, slightly earlier than that of Tc ct. Interestingly, in D. melanogaster, cato expression is only temporarily restricted to specific sense organ types. The gene is initially only expressed in the Ato- and Amos-dependent sense organs, but later expression appears also in the larval external mechanosensory sensilla, after $a c$-sc are switched off and after the start of $c t$ expression [34].

\section{Evolutionary scenario}

In the following, we discuss a model that explains how the mechanisms underlying sense organ diversification in arthropods might have evolved. Sensory genes are active at different time points and thus can be assigned different roles in the process of subtype identity development according to their expression and mutant phenotypes: (1) formation and subtype specification of SOPs, (2) specification of accessory (e.g. bristles, sockets) and neural cell types, and (3) differentiation of sense organ cells (Fig. 7).

In all arthropod species analysed, $A S H$ is at the top of the sensory gene cascade and shows a widespread early expression in different morphological and functional types of sensilla prior to or during formation of the SOPs $[9,40,69]$. We therefore suggest that in the last common ancestor of arthropods, $A S H$ was the predominant proneural gene for sense organ development and that ASH endowed epidermal cells with the potential to develop into external sense organs without simultaneously specifying subtype identity (Fig. 7a). The presence of an Ato-like gene in prebilaterian sense organs [70] suggests that ato belonged to the sensory toolkit of the arthropod ancestor together with 'general' cell fate specification and differentiation genes that are expressed in all sense organs in all arthropod species that have been analysed so far (sna, pros, $N$, nb; e.g. $[9,39,40,69]$ ). Since members of both protostomian phyla (Ecdysozoa and Lophotrochozoa) possess functionally diverse sense organs (e.g. $[10,71])$, we can assume that the last common ancestor of arthropods started out with some diversity of sensory neurons and accessory cells (Fig. 7a). Based on studies in arthropod groups other than insects, the crustaceans, myriapods, and chelicerates, we can furthermore assume that the ancestral sense organs in the last common ancestor of the euarthropods developed from groups of SOPs, rather than single SOP lineages as seen in dipterans $[9,40,69]$.

Initially, genes that were added to sensory development might have appeared late in development and were required for regulating neuronal differentiation such as axonal projections and the structure of dendrites (Fig. 7a). The increase of the sensory gene toolkit might have been supported by lineage-specific duplications of 


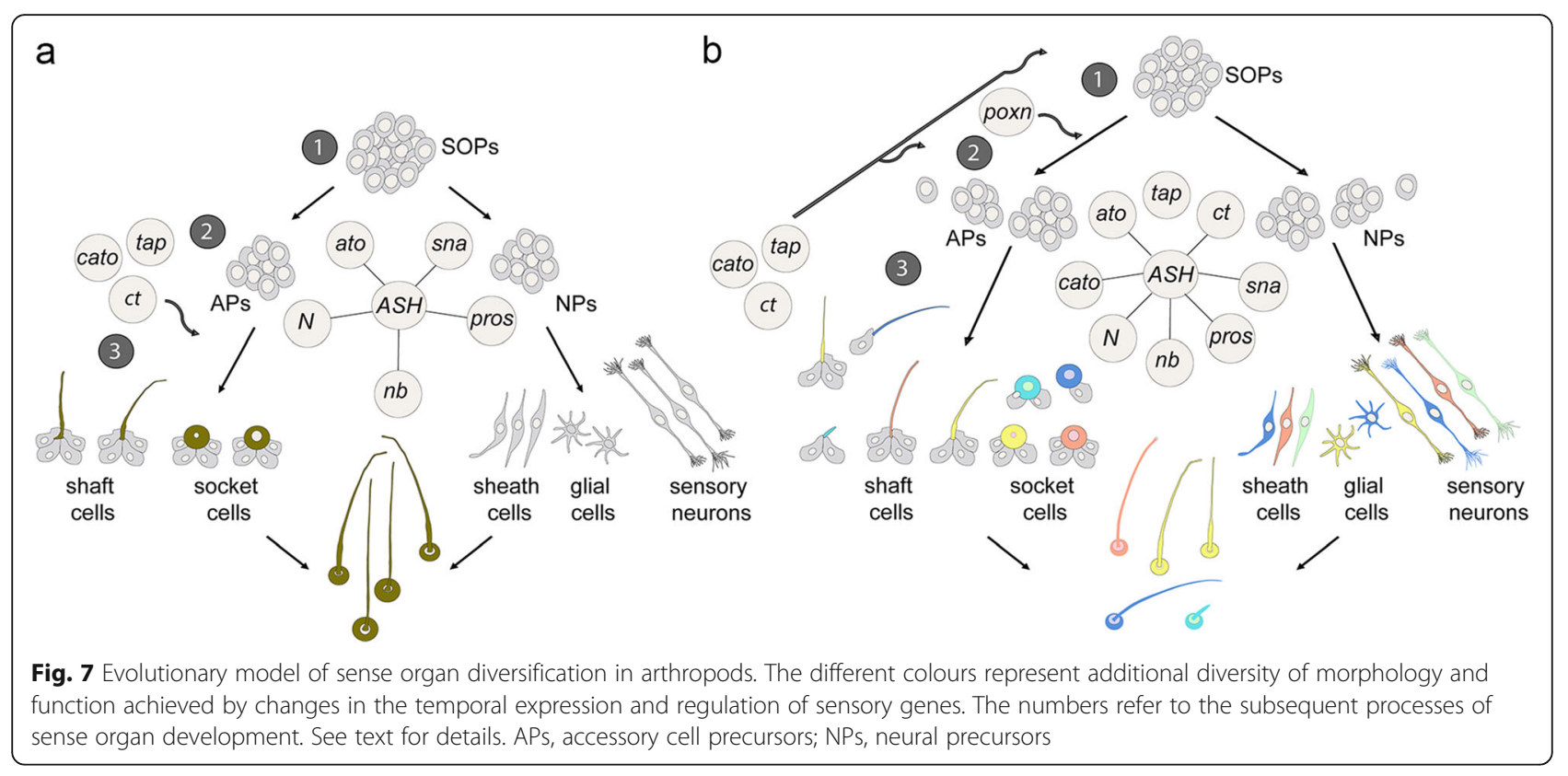

genes already part of the network, such as ASH and Atonal family members $[27,34,72]$. An example is the lineage-specific expansion of atonal genes in mosquitoes who harbour three atonal genes in their genome in contrast to most other insects [73]. In the next step, changes in the temporal expression of the add-on genes changed their role and importance in sense organ development (Fig. 7b). Other genes might have been directly recruited to the earlier process of accessory (socket, shaft, sheath cells) and neuronal cell type specification (neurons, glia) to regulate the developmental potential and divisions of the secondary precursors (e.g. poxn). The addition of sensory genes to the existing set resulted in variations in cell type numbers and morphology that were used as an evolutionary tool for sense organ diversification (Fig. 7b).

This evolutionary scenario is supported by variations in the roles, regulation, and temporal expression of sensory genes observed in different sense organs and species. tap, for example, which is expressed late during the development of a subset of sense organs in $T$. castaneum and in gustatory sense organs in D. melanogaster, is expressed at the time of SOP formation in the D. melanogaster adult antennal olfactory sensilla [31]. Similarly, D. melanogaster cato shows variations in its temporal expression, ranging from SOP formation to terminal differentiation in different chordotonal organs and ESOs $[74,75]$. Furthermore, $\mathrm{Ct}$, which is expressed in ESO SOPs, has a late role in dendritic arborisation in a subtype of multidendritic (MD) neurons [76]. These examples indicate that the sensory gene network is modified locally by spatial and temporal cues, which has indeed been demonstrated in $D$. melanogaster (e.g. $[37,77,78]$ ). Similar context-dependent mechanisms might operate in
T. castaneum. For example, the Tc ASH-Tc ato positive sense organs, which occupy the same position directly opposite the tracheal pits in all trunk segments, develop into the gustatory plCSMs in the thoracic segments but into the olfactory plTSOs in the abdominal segments (Fig. 2d). The potential for spatial and temporal modulation of sense organ development opens up an additional evolutionary route for generating sensory diversity and provides an explanation for the lack of sense organ subtype specificity of the genes analysed here: proneural genes might be used interchangeably for different subtypes because their target gene specificity is modulated by positional cues. Thus, a conserved sensory gene network can be used flexibly in different contexts to generate diversity.

Further evolution of sense organ specification leading to the $D$. melanogaster model, where sense organs develop along alternative pathways, might have been supported by changes in the regulation of the sensory genes. This assumption is supported by variations in the coexpression of genes in different arthropod groups and sense organs. In representatives of crustacean and myriapods $[9,40]$ and in $T$. castaneum (this study), coexpression of Tc ASH and Tc ato has been observed in many sense organs. In addition, $T c c t$ is co-expressed both with ato and $A S H$. In contrast in D. melanogaster, both Amos and Ato execute their subtype identity role by suppressing $a c-s c$ and $c t$, respectively [32, 37].

\section{How did the urbilaterian specify its sense organs?}

The proposed evolutionary model is based on comparative studies in arthropods and suggests that in their last common ancestor $A S H$ was the predominant proneural 
gene and that promotion of neurogenesis (i.e. generation of SOPs) was not directly coupled with subtype identity. Does this ancestral state apply to arthropods only or can similarities be traced further back to the last common ancestor of bilaterians, the urbilaterian?

ASH and Atonal-like genes are part of the developmental toolkit of sensory cells in prebilaterians (e.g. sponges: [79]; e.g. cnidarians: [70, 80, 81]). In cnidarians, an Atonal/Neurogenin homologue is expressed more widely and earlier than $A S H$ indicating that ASH is involved in differentiation of sensory cells, rather than determination of SOPs [70, 81]. Furthermore, Atonal/ Neurogenin is not the first neural gene to be expressed in the progenitors: it is expressed after SoxB [70], suggesting that the gene product is required for the execution of the sensory programme, rather than the initial neural cell fate determination. All three gene families are also expressed in sense organs of representatives of Protostomia and Deuterostomia (e.g. [70]), additionally confirming that they must have belonged to the sensory gene network of the urbilaterian.

Furthermore, the gene families were likely able to support distinct proneural activities in the urbilaterian. This is supported by previous studies that replace the D. melanogaster ato coding sequence (CDS) with the CDSs of Ato/Neurogenin members from across the metazoans (mouse, lancelet, annelid, sponge), including the CDSs of the D. melanogaster Achaete-Scute Complex as outgroup [82]. While the Ato members and the Ato/Neurogenin sponge gene could rescue the $D$. melanogaster ato mutant sense organ phenotype, the neurogenins could not replace $D$. melanogaster ato function and neither could the members of the Achaete-Scute Complex.

A comparison of the role and expression of vertebrate proneural genes together with the data from prebilaterians [70] agrees with our model of generating diversity by shifts in the temporal expression of sensory genes. For example in vertebrates, cranial ganglion sensory neurons are specified by the ato-related gene NeuroG [83], while in the olfactory epithelium of the mouse, Ascl1 (formerly Mash1) is expressed first, followed by Math4C/neurogenin [84]. In both cases, NeuroD, another atonal-like gene, is expressed during differentiation of the sensory neurons. In the otic placode, neurogenin 1 is expressed at the top of the hierarchy in the neural progenitor and then in the sensory neuron together with NeuroD, followed by Math1 expression in the mechanosensory hair cell, a distinct ear cell type [85, 86]. These data are again in line with our findings in the T. castaneum showing that proneural families can support diverse sensory functions and morphologies.

Furthermore, it can be assumed that the function of proneural genes of simultaneously promoting neural fate and subtype identity was uncoupled in the urbilaterian.
This assumption is in line with the data presented here and supported by the mechanisms of sensory neuron specification in the sensory placodes of vertebrates. For example, in the olfactory placode, proneural genes are not expressed in the olfactory stem cells, which give rise to diverse neurons; rather, Ascl1 is expressed in the socalled transit amplifying neuronal progenitors that have progressed from the multipotent precursor state and the gene is required for the expansion of the olfactory receptor neurons [87]. Subtype identity of the different olfactory neuron populations (olfactory receptor neurons, vomeronasal receptor neurons, and gonadotropin releasing hormone neurons) is achieved by inductive signalling from the underlying mesenchyme (e.g. [88, 89]).

\section{Conclusions}

Taken together, our results support an evolutionary scenario whereby sensory genes are recruited to the development of individual or subsets of sense organs, which do not necessarily fall into specific morphological or functional classes. Changes in the temporal expression can move the genes up in the hierarchy so that they can control all aspects of a specific sense organ subtype, as is the case for $c t$ and poxn in D. melanogaster, for example. The $T$. castaneum data presented here also fit this evolutionary scenario. Tc $c t$ has been recruited to a late step of development in all sense organs, regulating the differentiation of shaft cells, while Tc Poxn is only required in a subset of sensilla possibly controlling cell type numbers. Tc cato is expressed earlier than Tc cut and might therefore be involved in sensory organ cell type specification, similar to Tc poxn but in all sense organs. None of these genes are expressed during formation of the SOPs and therefore do not have control over the whole specification process, i.e. sense organ subtype identity.

To conclude, the evolutionary scenario presented here suggests that sense organ subtype identity has evolved by recruitment of a flexible sensory gene network to the different sense organ specification processes. A dominant role of these genes in subtype identity has evolved as a secondary effect of the function of these genes in individual or subsets of sense organs. Positional cues must have had a major influence on the evolution of the subtype identity, and corresponding spatial and temporal enhancers have indeed been identified in proneural genes, e.g. [90, 91]. Future comparative analysis will show how patterning mechanisms have shaped the evolution of sense organ diversity.

\section{Methods}

\section{Animal husbandry}

$T$. castaneum beetles were reared as previously described [47]. The San Bernadino (SB) wildtype strain was used for all experiments. 
Sequence analysis and cloning of $T$. castaneum genes Tc ASH, Tc ato, Tc poxn, Tc cato, Tc tap, and Tc amos gene sequences were obtained from the iBeetle-Base [92, 93]. A phylogenetic tree using ClustalW alignment of selected bHLH containing protein sequences (ato, cato, amos) was generated (see Additional file 1: Figure S6 [94], Table S7 for sequence accession numbers) to demonstrate correctness of annotation. Sequences were amplified from cDNA (synthesised from extracted RNA from different developmental stages with SuperScript III FirstStrand Synthesis System (Invitrogen) and cloned into pGEM $^{\circ}$-T Easy Vector (Promega) using standard cloning procedures (Additional file 1: Table S8 for primer information)). Sanger sequencing (performed by Eurofins) was used to confirm sequences and orientation of fragments in the plasmid.

\section{mRNA probe synthesis and in situ hybridisation}

Antisense mRNA probes for genes in this study were synthesised from their cloned sequences (as described above). The in vitro transcriptions using the T7 RNA polymerase and the DIG RNA labelling Mix (both Roche) were performed following the supplier's protocol. Colorimetric whole mount in situ hybridisation (NBT/ $\mathrm{BCIP}$ ) was performed as previously described using antiDIG antibody conjugated with Alkaline-Phosphatase (Roche) [95].

\section{RNA interference}

Parental RNAi (pRNAi) (Tc ASH, Tc ato, Tc poxn, Tc cato, Tc tap, Tc amos) and embryonic RNAi (eRNAi) (Tc ct) were performed to infer gene functions. Double-stranded RNA (dsRNA) for all genes was ordered from Eupheria Biotech (Dresden, Germany; based on sequences from the iBeetle-Base [92, 93]). For each gene, two non-overlapping fragments (NOF1 and NOF2) were injected (where NOF1 is the same as the iB-RNA fragments used in the iBeetle screen [49]; see Additional file 1: Table S9 [49] and Figure S7 for list of iB-RNA numbers and location). For pRNAi, female pupae were injected with $1 \mu \mathrm{l} / \mu \mathrm{g}$ dsRNA as previously described ([96-98] and 'The Beetle Book', http:// wwwuser.gwdg.de/ gbucher1/tribolium-castaneumbeetle-book1.pdf). Embryos were injected with $3 \mu \mathrm{l} / \mu \mathrm{g}$ dsRNA as previously described [99]. The protocol was adjusted slightly. Embryo preparation, mounting, and injection were performed as described [99]. However, after injection, the coverslips with the embryos were placed upside down onto the oxygen permeable membrane with 3 stacked coverslips used as bridges, and the intervening space was filled with halocarbon oil. This petri dish set-up was inverted and placed on a layer of $1 \%$ agarose gel to maintain humidity. The embryos were kept in this condition at $32{ }^{\circ} \mathrm{C}$ until the embryos hatched. For pRNAi and eRNAi experiments, negative controls were either injected with $\mathrm{H}_{2} \mathrm{O}$ or injection buffer $(1.4 \mathrm{mM} \mathrm{NaCl}, 0.07 \mathrm{mM}$ Na2HPO4, 0.03 mM KH2PO4, 4 mM KCl, pH 6.8).

\section{Quantification of RNAi phenotypes and statistical analyses}

First instar larvae (L1) of pRNAi and eRNAi experiments were used for cuticle analysis and prepared as described before [100]. L1 cuticle preparations were analysed for wildtype larvae (wt), 'phenotype', 'non-specific' (i.e. broken cuticles, larvae still in egg membrane and hence sensilla not analysable, etc.), and 'empty eggs' (results are summarised in Additional file 1: Table S6). The larvae showing a phenotype were screened for the sensilla in prominent positions described in Fig. 1. These sensilla were counted in each larva on one side (preferably right side) and categorised, where applicable, as 'wildtype', 'missing sensilla', 'duplicated sensilla', 'reduced length of sensillum shaft', and 'only socket of sensillum present'. The recorded data for each gene are summarised for dsRNA NOF1 and NOF2, and further categorised into sensilla subtypes (TSOs, BSMs, CSMs, and CSGs) and body parts (head, thorax, abdomen). An overview of the recorded data can be found in Additional file 1: Tables S1-5. Microsoft Excel was used to document and process data for statistical analysis.

\section{SEM sample preparation}

T. castaneum 1 st instar larvae $(24 \mathrm{~h}$ egg lays were incubated at $32{ }^{\circ} \mathrm{C}$ for 3 days) were collected and washed with PBS. The washed larvae were fixed in 1 : 1 heptane and $3 \%$ glutaraldehyde at room temperature for $1 \mathrm{~h}$. The fixative was removed, and the larvae were dehydrated with a series of acetone $(70 \%, 80 \%, 2 \times 90 \%, 2 \times 100 \%)$. HMDS was used to dry the larvae (larvae were incubated in HMDS, and then, the solution was removed, followed by air drying larvae in a block dish overnight under the fume hood). The dried larvae were mounted on aluminium stubs with sticky tape and sputter coated with gold (Agar auto sputter coater model 108A). A FEI Quanta 3DFEG or a FEI Inspect $F$ electron microscope was used for imaging L1 larvae visualising sensilla morphology.

\section{Microscopy and image processing}

Following in situ hybridisation (colorimetric), the yolk of T. castaneum embryos was removed. The embryos were then mounted flat onto microscope slides using glycerol. Colorimetric stained embryos (NBT/BCIP staining reaction) as well as cleared cuticles of $T$. castaneum larvae were imaged, or screened using an inverse Leica microscope (DM IL, Wetzlar, Germany) and corresponding LAS software (version 2.8.1). Image acquisition of L1 cuticles was performed with a Leica SP5 confocal 
microscope (Wetzlar, Germany) and corresponding LAS $\mathrm{X}$ software. Z-stacks of L1 confocal images were processed using the Z-projection tool of Fiji [101]. Graphic design programs (Adobe photoshop CS2, Adobe illustrator, and Inkscape version 0.92) were used for image processing, assembly, and preparations of schematic illustrations.

\section{Supplementary Information}

The online version contains supplementary material available at https://doi. org/10.1186/s12915-021-00948-y.

Additional file 1: 'Functional analysis of sense organ specification in the Tribolium castaneum larva reveals divergent mechanisms in insects': Supplementary figures and tables. The file contains Figures S1-S7 and Tables S1-S9 in pdf format.

\section{Acknowledgements}

We thank Elke Küser, Claudia Hinners, and Gregor Bucher for providing SB Tribolium castaneum beetles and for permitting the use of the injection equipment at the Georg-August University in Göttingen and Vicente AraulloPeters from the NanoVison Centre at QMUL for support taking SEM images.

\section{Authors' contributions}

MIS and MK contributed equally to the experimental work. MAB performed part of the functional experiments. AS designed the project and prepared the manuscript. All authors contributed to writing the manuscript. All authors read and approved the final manuscript.

\section{Funding}

This work was supported by Queen Mary University of London studentships to MIS and MK. The funders had no role in the design of the study and collection, analysis, and interpretation of data and in writing the manuscript.

\section{Availability of data and materials}

All data generated or analysed during this study are included in this published article and its supplementary information files.

\section{Ethics approval and consent to participate}

Not applicable

\section{Consent for publication}

Not applicable

\section{Competing interests}

The authors declare that they have no competing interests.

\section{Author details}

'School of Biological and Chemical Sciences, Queen Mary University of London, Mile End Road, London E1 4NS, UK. ' Marine Eco-Evo-Devo Unit, Okinawa Institute for Science and Technology (OIST), 1919-1 Tancha, Onna-son, Okinawa 904-0495, Japan. ${ }^{3}$ Department of Zoology, University of Cambridge, Downing St, Cambridge CB2 3EJ, UK

Received: 21 October 2020 Accepted: 4 January 2021

Published online: 05 February 2021

\section{References}

1. Barth FG. A spider's world - senses and behavior: Springer; 2001 www.springer.com/de/book/9783540420460. Accessed 20 Nov 2017

2. Dangles O, Irschick D, Chittka L, Casas J. Variability in sensory ecology: expanding the bridge between physiology and evolutionary biology. Q Rev Biol. 2009;84:51-74.

3. Hansson BS, Stensmyr MC. Evolution of insect olfaction. Neuron 2011:72: 698-711.

4. Stevens M. Sensory ecology, behaviour, and evolution. Oxford: Oxford University Press; 2013.
5. Shanbhag SR, Müller B, Steinbrecht RA. Atlas of olfactory organs of Drosophila melanogaster: 1. Types, external organization, innervation and distribution of olfactory sensilla. Int J Insect Morph Embryol. 1999;28:377-97.

6. Dahanukar A, Hallem EA, Carlson JR. Insect chemoreception. Curr Opin Neurobiol. 2005;15:423-30.

7. Joseph RM, Carlson JR. Drosophila chemoreceptors: a molecular interface between the chemical world and the brain. Trends Genet. 2015;31:683-95.

8. Chapman RF, Simpson SJ, Douglas AE. The insects: structure and function. New York: Cambridge University Press; 2013

9. Klann M, Stollewerk A. Evolutionary variation in neural gene expression in the developing sense organs of the crustacean Daphnia magna. Dev Biol. 2017:424:50-61.

10. Hartenstein V. Development of insect sensilla. In: Comprehensive molecular insect science: Elsevier; 2005. p. 379-419. https://doi.org/10.1016/B0-44451924-6/00012-0.

11. Keil TA. Functional morphology of insect mechanoreceptors. Microsc Res Tech. 1997:39:506-31.

12. Snodgrass RE. The morphology of insect sense organs and the sensory nervous system. Smithson Miscellaneous Collections. 1926;77:1-82.

13. Stocker RF. The organization of the chemosensory system in Drosophila melanogaster: a rewiew. Cell Tissue Res. 1994;275:3-26.

14. Hallberg E, Hansson BS. Arthropod sensilla: morphology and phylogenetic considerations. Microsc Res Tech. 1999;47:428-39.

15. Ryan MF, Behan M. The sensory receptors of Tribolium larvae. Physiol Zool. 1973;46:238-44

16. Ploomi A, Merivee E, Rahi M, Bresciani J, Ravn HP, Luik A, et al. Antennal sensilla in ground beetles (Coleoptera: Carabidae). Agron Res. 2003;1:221-8.

17. Jarman AP, Grau Y, Jan LY, Jan YN. atonal is a proneural gene that directs chordotonal organ formation in the Drosophila peripheral nervous system. Cell. 1993;73:1307-21.

18. Bertrand N, Castro DS, Guillemot F. Proneural genes and the specification of neural cell types. Nat Rev Neurosci. 2002:3:517-30.

19. Lai EC, Orgogozo V. A hidden program in Drosophila peripheral neurogenesis revealed: fundamental principles underlying sensory organ diversity. Dev Biol. 2004;269:1-17

20. Maung SMTW, Jarman AP. Functional distinctness of closely related transcription factors: a comparison of the Atonal and Amos proneural factors. Mech Dev. 2007:124:647-56.

21. González F, Romani S, Cubas P, Modolell J, Campuzano S. Molecular analysis of the asense gene, a member of the achaete-scute complex of Drosophila melanogaster, and its novel role in optic lobe development. EMBO J. 1989;8: 3553-62.

22. Doe CQ, Chu-LaGraff Q, Wright DM, Scott MP. The prospero gene specifies cell fates in the Drosophila central nervous system. Cell. 1991;65:451-64.

23. Domínguez M, Campuzano S. asense, a member of the Drosophila achaetescute complex, is a proneural and neural differentiation gene. EMBO J. 1993; 12:2049-60

24. Brand M, Jarman AP, Jan LY, Jan YN. asense is a Drosophila neural precursor gene and is capable of initiating sense organ formation. Development. 1993;119:1-17.

25. Jarman AP, Brand $M$, Jan $L Y$, Jan $Y N$. The regulation and function of the helix-loop-helix gene, asense, in Drosophila neural precursors. Development. 1993;119:19-29.

26. Ip YT, Levine M, Bier E. Neurogenic expression of snail is controlled by separable CNS and PNS promoter elements. Development. 1994;120:199_ 207.

27. Ayyar S, Negre B, Simpson P, Stollewerk A. An arthropod cis-regulatory element functioning in sensory organ precursor development dates back to the Cambrian. BMC Biol. 2010:8:127

28. Johnson NAN, Wang Y, Zeng Z, Wang G-D, Yao Q, Chen K-P. Phylogenetic analysis and classification of insect achaete-scute complex genes. J Asia Pac Entomol. 2019:22:398-403.

29. Nottebohm E, Usui A, Therianos S, Kimura K, Dambly-Chaudiére C, Ghysen A. The gene poxn controls different steps of the formation of chemosensory organs in Drosophila. Neuron. 1994;12:25-34

30. Gautier P, Ledent V, Massaer M, Dambly-Chaudière C, Ghysen A. tap, a Drosophila bHLH gene expressed in chemosensory organs. Gene. 1997;191: $15-21$.

31. Ledent V, Gaillard F, Gautier P, Ghysen A, Dambly-Chaudière C. Expression and function of tap in the gustatory and olfactory organs of Drosophila. Int J Dev Biol. 1998;42:163-70. 
32. Jarman AP, Ahmed I. The specificity of proneural genes in determining Drosophila sense organ identity. Mech Dev. 1998;76:117-25.

33. Goulding SE, White NM, Jarman AP. cato encodes a basic helix-loop-helix transcription factor implicated in the correct differentiation of Drosophila sense organs. Dev Biol. 2000;221:120-31.

34. Goulding SE, zur Lage P, Jarman AP. amos, a proneural gene for Drosophila olfactory sense organs that is regulated by lozenge. Neuron. 2000;25:69-78.

35. Brewster R, Hardiman K, Deo M, Khan S, Bodmer R. The selector gene cut represses a neural cell fate that is specified independently of the AchaeteScute-Complex and atonal. Mech Dev. 2001;105:57-68.

36. Skaer N, Pistillo D, Gibert J-M, Lio P, Wülbeck C, Simpson P. Gene duplication at the achaete-scute complex and morphological complexity of the peripheral nervous system in Diptera. Trends Genet. 2002;18:399-405.

37. zur Lage PI, Prentice DRA, Holohan EE, Jarman AP. The Drosophila proneural gene amos promotes olfactory sensillum formation and suppresses bristle formation. Development. 2003;130:4683-93.

38. Bodmer R, Barbel S, Sheperd S, Jack JW, Jan LY, Jan YN. Transformation of sensory organs by mutations of the cut locus of D. melanogaster. Cell. 1987; 51:293-307.

39. Gold K, Cotton JA, Stollewerk A. The role of Notch signalling and numb function in mechanosensory organ formation in the spider Cupiennius salei. Dev Biol. 2009;327:121-31.

40. Pioro HL, Stollewerk A. The expression pattern of genes involved in early neurogenesis suggests distinct and conserved functions in the diplopod Glomeris marginata. Dev Genes Evol. 2006;216:417-30.

41. Schinko JB, Kreuzer N, Offen N, Posnien N, Wimmer EA, Bucher G. Divergent functions of orthodenticle, empty spiracles and buttonhead in early head patterning of the beetle Tribolium castaneum (Coleoptera). Dev Biol. 2008; 317:600-13.

42. Behan M, Ryan MF. Ultrastructure of antennal sensory receptors of Tribolium larvae (Coleoptera: Tenebrionidae). Int J Insect Morph Embryol. 1978;7:22136.

43. Biffar L, Stollewerk A. Conservation and evolutionary modifications of neuroblast expression patterns in insects. Dev Biol. 2014;388:103-16.

44. Wheeler SR, Carrico ML, Wilson BA, Brown SJ, Skeath JB. The expression and function of the achaete-scute genes in Tribolium castaneum reveals conservation and variation in neural pattern formation and cell fate specification. Development. 2003;130:4373-81.

45. Savard J, Marques-Souza H, Aranda M, Tautz D. A segmentation gene in Tribolium produces a polycistronic mRNA that codes for multiple conserved peptides. Cell. 2006;126:559-69.

46. Benton MA, Akam M, Pavlopoulos A. Cell and tissue dynamics during Tribolium embryogenesis revealed by versatile fluorescence labeling approaches. Development. 2013;140:3210-20.

47. Brown SJ, Shippy TD, Miller S, Bolognesi R, Beeman RW, Lorenzen MD, et al. The red flour beetle, Tribolium castaneum (Coleoptera): a model for studies of development and pest biology. Cold Spring Harb Protocol. 2009;2009: pdb.emo126.

48. Tomoyasu Y, Denell RE. Larval RNAi in Tribolium (Coleoptera) for analyzing adult development. Deve Genes Evol. 2004;214:575-8.

49. Schmitt-Engel C, Schultheis D, Schwirz J, Ströhlein N, Troelenberg N, Majumda $U$, et al. The iBeetle large-scale RNAi screen reveals gene functions for insect development and physiology. Nat Commun. 2015;6:7822.

50. Finet C, Decaras A, Armisén D, Khila A. The achaete-scute complex contains a single gene that controls bristle development in the semi-aquatic bugs. Proc Royal Soc B Biol Sci. 2018;285:20182387.

51. Grillenzoni N, de Vaux V, Meuwly J, Vuichard S, Jarman A, Holohan E, et al. Role of proneural genes in the formation of the larval olfactory organ of Drosophila. Dev Genes Evol. 2007;217:209-19.

52. de Miguel C, Linsler F, Casanova J, Franch-Marro X. Genetic basis for the evolution of organ morphogenesis: the case of spalt and cut in the development of insect trachea. Development. 2016;143:3615-22.

53. Lovegrove B, Simões S, Rivas ML, Sotillos S, Johnson K, Knust E, et al. Coordinated control of cell adhesion, polarity, and cytoskeleton underlies hox-induced organogenesis in Drosophila. Curr Biol. 2006;16:2206-16.

54. Hanna L, Popadić A. A hemipteran insect reveals new genetic mechanisms and evolutionary insights into tracheal system development. PNAS. 2020; 117:4252-61.

55. Campuzano S, Carramolino L, Cabrera CV, Ruiz-Gómez M, Villares R, Boronat $A$, et al. Molecular genetics of the achaete-scute gene complex of $D$. melanogaster. Cell. 1985;40:327-38.
56. Cubas P, Modolell J. The extramacrochaetae gene provides information for sensory organ patterning. EMBO J. 1992;11:3385-93.

57. Reddy GV, Gupta B, Ray K, Rodrigues V. Development of the Drosophila olfactory sense organs utilizes cell-cell interactions as well as lineage. Development. 1997;124:703-12

58. Marcellini S, Gibert J-M, Simpson P. achaete, but not scute, is dispensable for the peripheral nervous system of Drosophila. Dev Biol. 2005;285:545-53.

59. Huang F, Dambly-Chaudiere C, Ghysen A. The emergence of sense organs in the wing disc of Drosophila. Development. 1991;111:1087-95.

60. Gupta BP, Rodrigues V. atonal is a proneural gene for a subset of olfactory sense organs in Drosophila. Genes Cells. 1997;2:225-33.

61. Rodríguez I, Hernández R, Modolell J, Ruiz-Gómez M. Competence to develop sensory organs is temporally and spatially regulated in Drosophila epidermal primordia. EMBO J. 1990;9:3583-92.

62. Hinz U, Giebel B, Campos-Ortega JA. The basic-helix-loop-helix domain of Drosophila lethal of scute protein is sufficient for proneural function and activates neurogenic genes. Cell. 1994;76:77-87.

63. Blochlinger $K$, Jan LY, Jan YN. Transformation of sensory organ identity by ectopic expression of Cut in Drosophila. Genes Dev. 1991;5:1124-35.

64. Awasaki T, Kimura K. pox-neuro is required for development of chemosensory bristles in Drosophila. J Neurobiol. 1997;32:707-21.

65. Merritt DJ. Transformation of external sensilla to chordotonal sensilla in the cut mutant of Drosophila assessed by single-cell marking in the embryo and larva. Microsc Res Tech. 1997;39:492-505.

66. Jiang $Y$, Boll W, Noll M. Pox neuro control of cell lineages that give rise to larval poly-innervated external sensory organs in Drosophila. Dev Biol. 2015; 397:162-74.

67. Dambly-Chaudière C, Jamet E, Burri M, Bopp D, Basler K, Hafen E, et al. The paired box gene pox neuro: a determinant of poly-innervated sense organs in Drosophila. Cell. 1992;69:159-72

68. Hartenstein V. Development of Drosophila larval sensory organs: spatiotemporal pattern of sensory neurones, peripheral axonal pathways and sensilla differentiation. Development. 1988;102:869-86.

69. Stollewerk A, Seyfarth E-A. Evolutionary changes in sensory precursor formation in arthropods: embryonic development of leg sensilla in the spider Cupiennius salei. Dev Biol. 2008;313:659-73.

70. Richards GS, Rentzsch F. Regulation of Nematostella neural progenitors by SoxB, Notch and bHLH genes. Development. 2015;142:3332-42.

71. Wright KA. Peripheral sensilla of some lower invertebrates: the Platyhelminthes and Nematoda. Microsc Res Tech. 1992;22:285-97.

72. Jarman AP, Groves AK. The role of Atonal transcription factors in the development of mechanosensitive cells. Sem Cell Dev Biol. 2013;24:438-47.

73. Zhang DB, Wang Y, Liu AK, Wang XH, Dang CW, Yao Q, et al. Phylogenetic analyses of vector mosquito basic helix-loop-helix transcription factors. Insect Mol Biol. 2013;22:608-21.

74. Blochlinger $K$, Bodmer R, Jan LY, Jan YN. Patterns of expression of cut, a protein required for external sensory organ development in wild-type and cut mutant Drosophila embryos. Genes Dev. 1990:4:1322-31.

75. zur Lage PI, Jarman AP. The function and regulation of the bHLH gene, cato, in Drosophila neurogenesis. BMC Dev Biol. 2010;10:34.

76. Ebacher DJS, Todi SV, Eberl DF, Boekhoff-Falk GE. cut mutant Drosophila auditory organs differentiate abnormally and degenerate. Fly. 2007;1:86-94.

77. Rozowski M, Akam M. Hox gene control of segment-specific bristle patterns in Drosophila. Genes Dev. 2002;16:1150-62.

78. Eksi SE, Barmina O, McCallough CL, Kopp A, Orenic TV. A Distallessresponsive enhancer of the Hox gene Sex combs reduced is required for segment- and sex-specific sensory organ development in Drosophila. PLoS Genet. 2018;14:e1007320.

79. Mah JL, Leys SP. Think like a sponge: the genetic signal of sensory cells in sponges. Dev Biol. 2017;431:93-100.

80. Galliot B, Quiquand M, Ghila L, de Rosa R, Miljkovic-Licina M, Chera S. Origins of neurogenesis, a cnidarian view. Dev Biol. 2009;332:2-24.

81. Grens A, Mason E, Marsh JL, Bode HR. Evolutionary conservation of a cell fate specification gene: the Hydra achaete-scute homolog has proneural activity in Drosophila. Development. 1995;121:4027-35.

82. Weinberger S, Topping MP, Yan J, Claeys A, Geest ND, Ozbay D, et al. Evolutionary changes in transcription factor coding sequence quantitatively alter sensory organ development and function. eLife. 2017;6:e26402.

83. Castro DS, Guillemot F. Old and new functions of proneural factors revealed by the genome-wide characterization of their transcriptional targets. Cell Cycle. 2011;10:4026-31. 
84. Cau E, Gradwohl G, Fode C, Guillemot F. Mash1 activates a cascade of bHLH regulators in olfactory neuron progenitors. Development. 1997;124:1611-21.

85. Fritzsch B, Beisel KW, Bermingham NA. Developmental evolutionary biology of the vertebrate ear: conserving mechanoelectric transduction and developmental pathways in diverging morphologies. NeuroReport. 2000;11: R35.

86. Fritzsch B, Elliott KL. Gene, cell, and organ multiplication drives inner ear evolution. Dev Biol. 2017;431:3-15.

87. Tucker ES, Lehtinen MK, Maynard T, Zirlinger M, Dulac C, Rawson N, et al. Proliferative and transcriptional identity of distinct classes of neural precursors in the mammalian olfactory epithelium. Development. 2010;137: 2471-81.

88. Rawson NE, Lischka FW, Yee KK, Peters AZ, Tucker ES, Meechan DW, et al. Specific mesenchymal/epithelial induction of olfactory receptor, vomeronasal, and gonadotropin-releasing hormone $(\mathrm{GnRH})$ neurons. Dev Dyn. 2010;239:1723-38

89. Parkash J, Cimino I, Ferraris N, Casoni F, Wray S, Cappy H, et al. Suppression of $\beta 1$-integrin in gonadotropin-releasing hormone cells disrupts migration and axonal extension resulting in severe reproductive alterations. J Neurosci. 2012;32:16992-7002.

90. Biryukova I, Heitzler P. The Drosophila LIM-homeodomain protein Islet antagonizes proneural cell specification in the peripheral nervous system. Dev Biol. 2005;288:559-70.

91. Niwa N, Hiromi Y, Okabe M. A conserved developmental program for sensory organ formation in Drosophila melanogaster. Nat Genet. 2004;36: ng1308.

92. Dönitz J, Schmitt-Engel C, Grossmann D, Gerischer L, Tech M, Schoppmeier $M$, et al. iBeetle-Base: a database for RNAi phenotypes in the red flour beetle Tribolium castaneum. Nucleic Acids Res. 2015;43:D720-5.

93. Dönitz J, Gerischer L, Hahnke S, Pfeiffer S, Bucher G. Expanded and updated data and a query pipeline for iBeetle-Base. Nucleic Acids Res. 2018;46:D831-5.

94. Price MN, Dehal PS, Arkin AP. FastTree: computing large minimum evolution trees with profiles instead of a distance matrix. Mol Biol Evol. 2009;26:164150 .

95. Schinko J, Posnien N, Kittelmann S, Koniszewski N, Bucher G. Single and double whole-mount In situ hybridization in red flour beetle (Tribolium) embryos. Cold Spring Harb Protocol. 2009:2009:pdb.prot5258.

96. Bucher G, Scholten J, Klingler M. Parental RNAi in Tribolium (Coleoptera). Curr Biol. 2002;12:R85-6.

97. Bucher $\mathrm{G}$, Klingler M. Divergent segmentation mechanism in the short germ insect Tribolium revealed by giant expression and function. Development. 2004;131:1729-40.

98. Posnien N, Schinko J, Grossmann D, Shippy TD, Konopova B, Bucher G. RNAi in the red flour beetle (Tribolium). Cold Spring Harb Protocol. 2009;2009: pdb.prot5256.

99. Benton MA. A revised understanding of Tribolium morphogenesis further reconciles short and long germ development. PLoS Biol. 2018;16:e2005093.

100. Wohlfrom H, Schinko JB, Klingler M, Bucher G. Maintenance of segment and appendage primordia by the Tribolium gene knödel. Mech Dev. 2006;123: 430-9.

101. Schindelin J, Arganda-Carreras I, Frise E, Kaynig V, Longair M, Pietzsch T, et al. Fiji: an open-source platform for biological-image analysis. Nat Methods. 2012;9:676-82.

\section{Publisher's Note}

Springer Nature remains neutral with regard to jurisdictional claims in published maps and institutional affiliations.

Ready to submit your research? Choose BMC and benefit from:

- fast, convenient online submission

- thorough peer review by experienced researchers in your field

- rapid publication on acceptance

- support for research data, including large and complex data types

- gold Open Access which fosters wider collaboration and increased citations

- maximum visibility for your research: over $100 \mathrm{M}$ website views per year

At BMC, research is always in progress.

Learn more biomedcentral.com/submissions 